Pure and Applied Mathematics Quarterly

Volume 4, Number 4

(Special Issue: In honor of

Jean-Pierre Serre, Part 1 of 2)

1133 - 1164,2008

\title{
$p$-adic Banach Modules of Arithmetical Modular Forms and Triple Products of Coleman's Families
}

\section{A. A. Panchishkin}

To dear Jean-Pierre Serre for his eightieth birthday with admiration

Abstract: For a prime number $p \geq 5$, we consider three classical cusp eigenforms

$$
f_{j}(z)=\sum_{n=1}^{\infty} a_{n, j} e(n z) \in \mathcal{S}_{k_{j}}\left(N_{j}, \psi_{j}\right), \quad(j=1,2,3)
$$

of weights $k_{1}, k_{2}, k_{3}$, of conductors $N_{1}, N_{2}, N_{3}$, and of nebentypus characters $\psi_{j} \bmod N_{j}$.

According to H.Hida [Hi86] and R.Coleman CoPB], one can include each $f_{j}$ $(j=1,2,3)$ (under suitable assumptions on $p$ and on $f_{j}$ )

$$
k_{j} \mapsto\left\{f_{j, k_{j}}=\sum_{n=1}^{\infty} a_{n}\left(f_{j, k_{j}}\right) q^{n}\right\}
$$

into a $p$-adic analytic family of cusp eigenforms $f_{j, k_{j}}$ of weights $k_{j}$ in such a way that $f_{j, k_{j}}=f_{j}$, and that all their Fourier coefficients $a_{n}\left(f_{j, k_{j}}\right)$ are given by certain $p$-adic analytic functions $k_{j} \mapsto a_{n, j}\left(k_{j}\right)$.

The conductor of the members of the families divides the prime-to- $p$ part of the level $N=\operatorname{LCM}\left(N_{1}, N_{2}, N_{3}\right)$.

The purpose of this paper is to describe a four variable $p$-adic $L$ function attached to Garrett's triple product of three Coleman's families

$$
k_{j} \mapsto\left\{f_{j, k_{j}}=\sum_{n=1}^{\infty} a_{n, j}(k) q^{n}\right\}
$$

Received July 26, 2006.

Based on a talk for the French-German Seminar in Lille on November 9, 2005 
of cusp eigenforms of three fixed slopes $\sigma_{j}=v_{p}\left(\alpha_{p, j}^{(1)}\left(k_{j}\right)\right) \geq 0$ where $\alpha_{p, j}^{(1)}=$ $\alpha_{p, j}^{(1)}\left(k_{j}\right)$ is an eigenvalue (which depends on $k_{j}$ ) of Atkin's operator $U=U_{p}$ acting on Fourier expansions by $U\left(\sum_{n \geq 0}^{\infty} a_{n} q^{n}\right)=\sum_{n \geq 0}^{\infty} a_{n p} q^{n}$.

Let us consider the product of three eigenvalues:

$$
\lambda=\lambda\left(k_{1}, k_{2}, k_{3}\right)=\alpha_{p, 1}^{(1)}\left(k_{1}\right) \alpha_{p, 2}^{(1)}\left(k_{2}\right) \alpha_{p, 3}^{(1)}\left(k_{3}\right)
$$

and assume that the slope of this product

$$
\sigma=v_{p}\left(\lambda\left(k_{1}, k_{2}, k_{3}\right)\right)=\sigma\left(k_{1}, k_{2}, k_{3}\right)=\sigma_{1}+\sigma_{2}+\sigma_{3}
$$

is constant and positive for all triplets $\left(k_{1}, k_{2}, k_{3}\right)$ in an appropriate $p$-adic neighbourhood of the fixed triplet of weights $\left(k_{1}, k_{2}, k_{3}\right)$. The each value $\sigma_{j}$ is fixed.

We consider the $p$-adic weight space $X$ containing all $\left(k_{j}, \psi_{j}\right)$. Our $p$-adic $L$-functions are Mellin transforms of certain measures with values in $\mathcal{A}$, where $\mathcal{A}=\mathcal{A}(\mathcal{B})$ denotes an affinoid algebra associated with an affinoid space $\mathcal{B}$ as in [CoPB], where $\mathcal{B}=\mathcal{B}_{1} \times \mathcal{B}_{2} \times \mathcal{B}_{3}$, is an affinoid neighbourhood around $\left(k_{1}, k_{2}, k_{3}\right) \in X^{3}$ (with a given integers $k_{j}$ and fixed Dirichlet characters $\left.\psi_{j} \bmod N\right)$.

We construct such a measure from higher twists of classical Siegel-Eisenstein series, which produce distributions with values in certain Banach $\mathcal{A}$-modules $\mathcal{M}=\mathcal{M}(N ; \mathcal{A})$ of triple modular forms with coefficients in the algebra $\mathcal{A}$.

Acknowledgement. A part of these results was exposed in a talk at the FrenchGerman Seminar in Lille and the author is grateful to V.Gritsenko for the invitation.

It is a great pleasure for me to thank S.Boecherer, C.-G.Schmidt and R.SchulzePillot for valuable discussions.

Details of computations and proofs will appear elsewhere (a joint article with S.Boecherer in preparation for a special volume in the Contemporary Math. series of the AMS).

\section{Contents}

1. Introduction

Why study $L$-values attached to modular forms?

2. Generalities on triple products

3. Statement of the problem

4. Arithmetical nearly holomorphic modular forms 
5. Siegel-Eisenstein series 1144

Arithmetical nearly holomorphic Siegel modular forms 1145

5.1. Algebraic differential operators of Maass and Shimura 1145

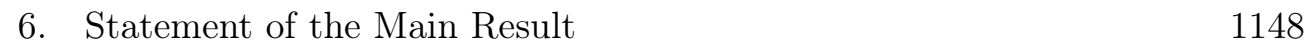

7. Distributions and admissible measures 1150

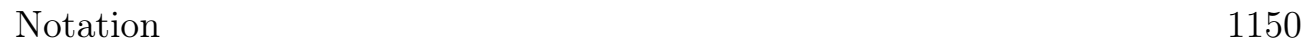

Admissible measures of Amice-Vélu 1151

7.1. $U_{p}-$ Operator and the method of canonical projection 1151

8. Triple modular forms 1152

Eigenfunctions of $U_{p}$ and of $U_{p}^{*}$. 1152

9. Critical values of the $L$ function $L\left(f_{1} \otimes f_{2} \otimes f_{3}, s, \chi\right) \quad 1153$

10. Theorems B-D 1154

11. Scheme of the Proof 1157

11.1. Boecherer's higher twist 1157

\begin{tabular}{lll}
\hline 11.2. & Ibukiyama's differential operator & 1157
\end{tabular}

11.3. Algebraic linear form 1158

11.4. Evaluation of $p$-adic integrals 1159

12. Criterion of admissibility 1160

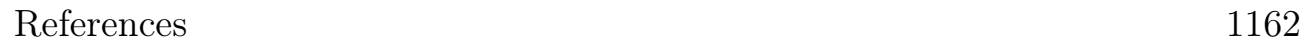

\section{INTRODUCTION}

Why study $L$-values attached to modular forms? A popular proceedure in Number Theory is the following: 
Construct a generating function $f=\sum_{n=0}^{\infty} a_{n} q^{n}$ $\in \mathbb{C}[[q]]$ of an arithmetical function $n \mapsto a_{n}$, for example $a_{n}=p(n)$

Example 1 Chand70:

(Hardy-Ramanujan)

$$
\begin{aligned}
& p(n)=\frac{e^{\pi \sqrt{2 / 3(n-1 / 24)}}}{4 \sqrt{3} \lambda_{n}^{2}} \\
& +O\left(e^{\pi \sqrt{2 / 3(n-1 / 24)}} / \lambda_{n}^{3}\right), \\
& \lambda_{n}=\sqrt{n-1 / 24},
\end{aligned}
$$

Compute $f$ via modular forms,

$$
\begin{aligned}
& \sum_{n=0}^{\infty} p(n) q^{n} \\
& =(\Delta / q)^{-1 / 24}
\end{aligned}
$$

Good bases, finite dimensions, many relations and identities

$$
\rightsquigarrow \begin{aligned}
& \text { A number } \\
& \text { (solution) }
\end{aligned}
$$

Values of $L$-functions, periods, congruences, ...

Other examples: Birch and Swinnerton-Dyer conjecture, $\ldots L$-values attached to modular forms

Our data: three primitive cusp eigenforms.

$$
f_{j}(z)=\sum_{n=1}^{\infty} a_{n, j} q^{n} \in \mathcal{S}_{k_{j}}\left(N_{j}, \psi_{j}\right), \quad(j=1,2,3)
$$

of weights $k_{1}, k_{2}, k_{3}$, of conductors $N_{1}, N_{2}, N_{3}$, and of nebentypus characters $\psi_{j} \bmod N_{j}, N=\operatorname{LCM}\left(N_{1}, N_{2}, N_{3}\right)$.

Let $p$ be a prime, $p \nmid N$. We view $f_{j} \in \overline{\mathbb{Q}} \llbracket q \rrbracket \stackrel{i_{p}}{\hookrightarrow} \mathbb{C}_{p} \llbracket q \rrbracket$ via a fixed embedding $\overline{\mathbb{Q}} \stackrel{i_{p}}{\hookrightarrow} \mathbb{C}_{p}, \mathbb{C}_{p}=\widehat{\overline{\mathbb{Q}}}_{p}$ is Tate's field.

Let $\chi$ denote a variable Dirichlet character $\bmod N p^{v}, v \geq 0$.

We view $k_{j}$ as a variable weight in the weight space $X=X_{N p^{v}}=\operatorname{Hom}_{\text {cont }}\left(Y, \mathbb{C}_{p}^{*}\right)$, $Y=(\mathbb{Z} / N \mathbb{Z})^{*} \times \mathbb{Z}_{p}^{*} \ni\left(y_{0}, y_{p}\right)$.

The space $X$ is a $p$-adic analytic space first used in Serre's Se73 "Formes modulaires et fonctions zêta $p$-adiques". Denote by $(k, \chi) \in X$ the homomorphism $\left(y_{0}, y_{p}\right) \mapsto \chi\left(y_{0}\right) \chi\left(y_{p} \bmod p^{v}\right) y_{p}^{k}$. We write simply $k_{j}$ for the couple $\left(k_{j}, \psi_{j}\right) \in X$.

The purpose of this paper is to describe a four variable p-adic L function attached to Garrett's triple product of three Coleman's families.

$$
k_{j} \mapsto\left\{f_{j, k_{j}}=\sum_{n=1}^{\infty} a_{n, j}\left(k_{j}\right) q^{n}\right\}
$$

of cusp eigenforms of three constant slopes $\sigma_{j}=\operatorname{ord}_{p}\left(\alpha_{p, j}^{(1)}\left(k_{j}\right)\right) \geq 0$ where $\alpha_{p, j}^{(1)}\left(k_{j}\right), \alpha_{p, j}^{(2)}\left(k_{j}\right)$ are the Satake parameters given as inverse roots of the Hecke $p$-polynomial $1-a_{p, j} X-\psi_{j}(p) p^{k_{j}-1} X^{2}=\left(1-\alpha_{p, j}^{(1)}(p) X\right)\left(1-\alpha_{p, j}^{(2)}(p) X\right)$. Each 
family of the associated primitive cusp eigenforms have conductors $C_{k_{j}}$ which divide $N$ if $k_{j}>2 \sigma_{j}+2$ (see [CoM] ).

We assume that $\operatorname{ord}_{p}\left(\alpha_{p, j}^{(1)}\left(k_{j}\right)\right) \leq \operatorname{ord}_{p}\left(\alpha_{p, j}^{(2)}\left(k_{j}\right)\right)$ and $\alpha_{p, j}^{(1)}\left(k_{j}\right) \neq \alpha_{p, j}^{(2)}\left(k_{j}\right)$

REMARK of the referee: In the elliptic modular case, an equality is expected to

never happen if the weight $>1$ (and this fact is proven when the weight $k$ is 2 and 3 by Coleman- Edixhoven and Ulmer under different conditions). However it happens in the Hilbert modular case, even when $k=2$. Anyway once we assume that $\alpha_{p, j}^{(1)} \neq \alpha_{p, j}^{(2)}$ for the initial weight, this holds in an open neighborhood of the weight.

The present paper extends a previous result: (see [PaTV]) where a two variable $p$-adic $L$-function was constructed interpolating on all $k$ a function $(k, s) \mapsto$ $L^{*}\left(f_{k}, s, \chi\right)(s=1, \cdots, k-1)$ for such a family.

We use the theory of $p$-adic integration with values in spaces of nearly holomorphic modular forms (in the sense of Shimura, see [ShiAr]).

A family of slope $\sigma>0$ of cusp eigenforms $f_{k}$ of weight $k \geq 2$ :

$$
\begin{array}{l|l}
k \mapsto f_{k}=\sum_{n=1}^{\infty} a_{n}(k) q^{n} & \begin{array}{l}
\text { 1) the Fourier coefficients } a_{n}(k) \text { of } f_{k} \\
\text { and one of the Satake }
\end{array} \\
\in \overline{\mathbb{Q}} \llbracket q \rrbracket \subset \mathbb{C}_{p} \llbracket q \rrbracket & \begin{array}{l}
p \text {-parameters } \alpha(k):=\alpha_{p}^{(1)}(k) \\
\text { A mode given by certain } p \text {-adic analytic } \\
\text { of a } p \text {-adic family } \\
\text { (not cusp and } \sigma=0):
\end{array} \\
\begin{array}{l}
\text { Eisenstein series } \\
a_{n}(k)=\sum_{d \mid n} d^{k-1}, f_{k}=E_{k}
\end{array} & \begin{array}{l}
\text { 2) the slope is constant and positive: } \\
\text { ord }(\alpha(k))=\sigma>0
\end{array}
\end{array}
$$

The existence of families of slope $\sigma>0$ was established in [CoPB]

R.Coleman gave an example with $p=7, f=\Delta, k=12$

$a_{7}=\tau(7)=-7 \cdot 2392, \sigma=1$.
A program in PARI for computing such families is discussed in CST98 (see also the Web-page of W.Stein, http://modular.fas.harvard.edu/ )

It was established by Coleman that: 
- The operator $U$ acts as a completely continuous operator on each $\mathcal{A}$-submodule $\mathcal{M}^{\dagger}\left(N p^{v} ; \mathcal{A}\right)$ $\subset \mathcal{A} \llbracket q \rrbracket$ (i.e. $U$ is a limit of finite-dimensional operators)

- there is a version of the Riesz theory: for any inverse root $\alpha \in \mathcal{A}^{*}$ of $P_{U}(T)$ there exists an eigenfunction $g, U g=\alpha g$
$\Longrightarrow$ there exists the Fredholm determinant $P_{U}(T)$ $=\operatorname{det}(I d-T \cdot U) \in \mathcal{A} \llbracket T \rrbracket$

such that $e v_{k}(g) \in \mathbb{C}_{p} \llbracket q \rrbracket$ are classical cusp eigenforms for all $k$ in a neigbourhood $\mathcal{B} \subset X$ (see in [CoPB])

We refer to $[\mathrm{CoPB}]$, part $\mathrm{B}$ and $[\mathrm{PaTV}]$, section 1, for generalities on rigid analytic $p$-adic families of modular forms.

\section{Generalities on triple products}

The triple product with a Dirichlet character $\chi$ is defined as the following complex $L$-function ( an Euler product of degree eight):

$$
L\left(f_{1} \otimes f_{2} \otimes f_{3}, s, \chi\right)=\prod_{p \nmid N} L\left(\left(f_{1} \otimes f_{2} \otimes f_{3}\right)_{p}, \chi(p) p^{-s}\right),
$$

$$
\begin{aligned}
& \text { where } L\left(\left(f_{1} \otimes f_{2} \otimes f_{3}\right)_{p}, X\right)^{-1}= \\
& \operatorname{det}\left(1_{8}-X\left(\begin{array}{cc}
\alpha_{p, 1}^{(1)} & 0 \\
0 & \alpha_{p, 1}^{(2)}
\end{array}\right) \otimes\left(\begin{array}{cc}
\alpha_{p, 2}^{(1)} & 0 \\
0 & \alpha_{p, 2}^{(2)}
\end{array}\right) \otimes\left(\begin{array}{cc}
\alpha_{p, 3}^{(1)} & 0 \\
0 & \alpha_{p, 3}^{(2)}
\end{array}\right)\right) \\
& =\prod_{\eta}\left(1-\alpha_{p, 1}^{(\eta(1))} \alpha_{p, 2}^{(\eta(2))} \alpha_{p, 3}^{(\eta(3))} X\right) \\
& =\left(1-\alpha_{p, 1}^{(1)} \alpha_{p, 2}^{(1)} \alpha_{p, 3}^{(1)} X\right)\left(1-\alpha_{p, 1}^{(1)} \alpha_{p, 2}^{(1)} \alpha_{p, 3}^{(2)} X\right) \cdot \ldots \cdot\left(1-\alpha_{p, 1}^{(2)} \alpha_{p, 2}^{(2)} \alpha_{p, 3}^{(2)} X\right),
\end{aligned}
$$

product taken over all 8 maps $\eta:\{1,2,3\} \rightarrow\{1,2\}$.

The Satake parameters and Hecke p-polynomials of forms $f_{j}$ : Here the Satake parameters $\alpha_{p, j}^{(1)}, \alpha_{p, j}^{(2)}$ are given as inverse roots of the Hecke $p$-polynomials

$$
1-a_{p, j} X-\psi_{j}(p) p^{k_{j}-1} X^{2}=\left(1-\alpha_{p, j}^{(1)}(p) X\right)\left(1-\alpha_{p, j}^{(2)}(p) X\right) .
$$

We always assume that the weights are "balanced":

$$
k_{1} \geq k_{2} \geq k_{3} \geq 2 \text {, and } k_{1} \leq k_{2}+k_{3}-2
$$

The non-balanced case (i.e. $k_{1}>k_{2}+k_{3}-2$ ) was treated in [HaTi], where $p$-adic measures for the square roots of special values of triple product $L$-functions, were constructed in the ordinary case. This construction can be probably extended to Coleman's case by techniques similar to the present paper. 
Critical values and functional equation. We use the corresponding normalized $L$ function (see [De79], [Co], Co-PeRi]), which has the form:

$$
\begin{aligned}
& \Lambda\left(f_{1} \otimes f_{2} \otimes f_{3}, s, \chi\right)= \\
& \Gamma_{\mathbb{C}}(s) \Gamma_{\mathbb{C}}\left(s-k_{3}+1\right) \Gamma_{\mathbb{C}}\left(s-k_{2}+1\right) \Gamma_{\mathbb{C}}\left(s-k_{1}+1\right) L\left(f_{1} \otimes f_{2} \otimes f_{3}, s, \chi\right),
\end{aligned}
$$

where $\Gamma_{\mathbb{C}}(s)=2(2 \pi)^{-s} \Gamma(s)$.

The Gamma-factor determines the critical values $s=k_{1}, \cdots, k_{2}+k_{3}-2$ of $\Lambda(s)$, which we explicitely evaluate (like in the classical formula $\zeta(2)=\frac{\pi^{2}}{6}$ ). A functional equation of $\Lambda(s)$ has the form:

$$
s \mapsto k_{1}+k_{2}+k_{3}-2-s .
$$

- According to H.Hida Hi86 and R.Coleman [CoPB], one can include each $f_{j}$ $(j=1,2,3)$ (under suitable assumptions on $p$ and on $\left.f_{j}\right)$ into a $p$-adic analytic family

$$
\mathbf{f}_{j}: k_{j} \mapsto\left\{f_{j, k_{j}}=\sum_{n=1}^{\infty} a_{n}\left(f_{j, k_{j}}\right) q^{n}\right\}
$$

of cusp eigenforms $f_{j, k_{j}}$ of weights $k_{j}$ in such a way that $f_{j, k_{j}}=f_{j}$, and that all their Fourier coefficients $a_{n}\left(f_{j, k_{j}}\right)$ are given by certain $p$-adic analytic functions $k_{j} \mapsto a_{n, j}\left(k_{j}\right)$.

\section{Statement of the Problem}

Given three $p$-adic analytic families $\mathbf{f}_{j}$ of slope $\sigma_{j} \geq 0$, to construct a four-variable $p$-adic $L$-function attached to Garrett's triple product of these families. We show that this function interpolates the special values

$$
\left(s, k_{1}, k_{2}, k_{2}\right) \longmapsto \Lambda\left(f_{1, k_{1}} \otimes f_{2, k_{2}} \otimes f_{3, k_{3}}, s, \chi\right)
$$

at critical points $s=k_{1}, \cdots, k_{2}+k_{3}-2$ for balanced weights $k_{1} \leq k_{2}+k_{3}-2$; we prove that these values are algebraic numbers after dividing out certain "periods".

However the construction uses directly modular forms, and not the $L$-values in question, and a comparison of special values of two functions is done after the construction.

Consider the product of the Satake parmeters

$$
\lambda_{p}=\alpha_{p, 1}^{(1)} \alpha_{p, 2}^{(1)} \alpha_{p, 3}^{(1)}=\lambda_{p}\left(k_{1}, k_{2}, k_{3}\right)
$$

We assume that $\operatorname{ord}_{p} \alpha_{p, j}^{(1)} \leq \operatorname{ord}_{p} \alpha_{p, j}^{(2)}$, and that the slope $\sigma=\operatorname{ord}_{p}\left(\lambda_{p}\left(k_{1}, k_{2}, k_{3}\right)\right)$ is constant and positive for all triplets $\left(k_{1}, k_{2}, k_{3}\right)$ in a $p$-adic neighbourhood $\mathcal{B} \subset X^{3}$ of the fixed triplet of weights $\left(k_{1}, k_{2}, k_{3}\right)$. 
Our method includes: - a version of Garrett's integral representation for the triple $L$-functions of the form: for $r=0, \cdots, k_{2}+k_{3}-k_{1}-2$,

$\Lambda\left(f_{1, k_{1}} \otimes f_{2, k_{2}} \otimes f_{3, k_{3}}, k_{2}+k_{3}-r, \chi\right)=$

$$
\iint_{\left.\Gamma_{0}\left(N^{2} p^{2 v}\right) \backslash \mathbb{H}\right)^{3}} \frac{\tilde{f}_{1, k_{1}}\left(z_{1}\right) \tilde{f}_{2, k_{2}}\left(z_{2}\right) \tilde{f}_{3, k_{3}}\left(z_{3}\right)}{\mathcal{E}}\left(z_{1}, z_{2}, z_{3} ;-r, \chi\right) \prod_{j}\left(\frac{d x_{j} d y_{j}}{y_{j}^{2}}\right)
$$

where $\tilde{f}_{j, k_{j}}=: f_{j, k_{j}}^{0}$ is an eigenfunction of $U_{p}^{*}$ in $\mathcal{M}_{k_{j}}\left(N p, \psi_{j}\right), f_{j, k_{j}, 0}$ is the corresponding eigenfunction of $U_{p}$, and

$\mathcal{E}\left(z_{1}, z_{2}, z_{3} ;-r, \chi\right) \in \mathcal{M}_{T}\left(N^{2} p^{2 v}\right)=\mathcal{M}_{k_{1}, r^{*}}\left(N^{2} p^{2 v}, \psi_{1}\right) \otimes \mathcal{M}_{k_{2}, r^{*}}\left(N^{2} p^{2 v}, \psi_{2}\right) \otimes \mathcal{M}_{k_{3}, r^{*}}\left(N^{2} p^{2 v}, \psi_{3}\right)$

is a (classical) nearly holomorphic triple modular form of triple weight $\left(k_{1}, k_{2}, k_{3}\right)$ of some type $r^{*} \geq 0$ (see [ShiAr]), and of fixed triple Nebentupus character $\left(\psi_{1}, \psi_{2}, \psi_{3}\right)$, obtained from a nearly holomorphic Siegel-Eisenstein series $F_{\chi, r}=$ $G^{\star}\left(z,-r ; k,\left(N p^{v}\right)^{2}, \boldsymbol{\psi}\right)$, of degree 3 , of weight $k=k_{2}+k_{3}-k_{1}$, and the Nebentypus character $\boldsymbol{\psi}=\chi^{2} \psi_{1} \psi_{2} \bar{\psi}_{3}$ ([Sh83]).

We obtain $\mathcal{E}\left(z_{1}, z_{2}, z_{3} ;-r, \chi\right)$ from a Siegel-Eisenstein series by applying to $F_{\chi, r}$ Boecherer's higher twist (see (11.22) ) and Ibukiyama's differential operator (see (11.23).

These operations act explicitely on the Fourier expansions. Then one uses:

- The theory of $p$-adic integration with values in Serre's type $\mathcal{A}$-modules $\mathcal{M}_{T}(\mathcal{A})$ of triple arithmetical nearly holomorphic modular forms over $p$-adic Banach algebras $\mathcal{A}$. We shall use the notation

$$
\mathcal{M}_{T}(\mathcal{A})=\mathcal{M}\left(\mathcal{A}\left(\mathcal{B}_{1}\right)\right) \hat{\otimes} \mathcal{M}\left(\mathcal{A}\left(\mathcal{B}_{2}\right)\right) \hat{\otimes} \mathcal{M}\left(\mathcal{A}\left(\mathcal{B}_{3}\right)\right)
$$

for certain $\mathcal{A}$-modules of $p$-adic families of triple modular forms.

Explicit Fourier coefficients $a_{\chi, r}(R, \mathcal{T}) \in \overline{\mathbb{Q}}[R, T]$ of $\mathcal{E}(-r, \chi)$ are given by special polynomials of matricies $\mathcal{T}=\left(t_{i j}\right), R=\left(R_{i j}\right)$ and of $\chi(\beta) \beta^{r}$ (with $\beta \in \mathbb{Z}_{p}^{*} \cap \mathbb{Q}$ ) i.e. the coefficients of $a_{\chi, r}$ by some elementry $p$-adic measures $\int_{Y} \chi y^{r} \mathrm{~d} \mu_{\mathcal{T}} \in \mathcal{A}$. Here $\mathcal{A}=\mathcal{A}(\mathcal{B})$ is a certain $p$-adic Banach algebra of functions on an open analytic subspace $\mathcal{B}=\mathcal{B}_{1} \times \mathcal{B}_{2} \times \mathcal{B}_{3} \subset X^{3}$ in the product of three copies of the weight space $X=\operatorname{Hom}_{\text {cont }}\left(Y, \mathbb{C}_{p}^{*}\right)$.

These measures on the group $Y=(\mathbb{Z} / N \mathbb{Z})^{*} \times \mathbb{Z}_{p}^{*}$ produce the coefficients of $a_{\chi, r}$ of $\mathcal{E}(-r, \chi)$ of $\mathcal{M}_{T}(\mathcal{A})$ for all $p$-adic weights $x \in X$, given by $\int_{Y} x(y) \mathrm{d} \mu_{\mathcal{T}} \in \mathcal{A}$ (an interpolation from $x=\chi y_{p}^{r}$ to all $x \in X$ ).

- The spectral theory of triple Atkin's operator $U=U_{p, T}$. allows to evaluate the integral using at each weight $\left(k_{1}, k_{2}, k_{3}\right)$ the equality $\left\langle\mathbf{f}^{0}, \mathcal{E}(-r, \chi)\right\rangle=\left\langle\mathbf{f}^{0}, \pi_{\lambda}(\mathcal{E}(-r, \chi))\right\rangle$ 
with the projection $\pi_{\lambda}$ of $\mathcal{M}_{T}(\mathcal{A})$ to the $\lambda$-part $\mathcal{M}_{T}(\mathcal{A})^{\lambda}$, defined by:

$$
\operatorname{Ker} \pi_{\lambda}:=\bigcap_{n \geq 1} \operatorname{Im}\left(U_{T}-\lambda I\right)^{n}, \quad \operatorname{Im} \pi_{\lambda}:=\bigcup_{n \geq 1} \operatorname{Ker}\left(U_{T}-\lambda I\right)^{n} .
$$

Note that

$$
\mathbf{f}^{0}=f_{k_{1}, 1}^{0} \otimes f_{k_{2}, 2}^{0} \otimes f_{k_{3}, 3}^{0}, \quad f_{k_{j}, j}^{0}=f_{k_{j}, j, 0}^{\rho} \mid k_{j}\left(\begin{array}{cc}
0 & -1 \\
N p & 0
\end{array}\right)(\text { for } j=1,2,3)
$$

is a classical form, defined at triple weghts $\left(k_{1}, k_{2}, k_{3}\right)$ as the Weil-involution image of $\mathbf{f}_{0}=f_{k_{1}, 1,0} \otimes f_{k_{2}, 2,0} \otimes f_{k_{3}, 3,0}$ as in [PaTV], at p.555. Note that the functions $f^{0}$ are eigenfunctions of the adjoint triple operator $U_{p}^{*}$. We consider also the sequences of triple modular forms:

$$
\mathbf{f}_{0}=\left\{f_{k_{1}, 1,0} \otimes f_{k_{2}, 2,0} \otimes f_{k_{3}, 3,0}\right\}_{\left(k_{1}, k_{2}, k_{3}\right)}, \mathbf{f}^{0}=\left\{f_{k_{1}, 1}^{0} \otimes f_{k_{2}, 2}^{0} \otimes f_{k_{3}, 3}^{0}\right\}_{\left(k_{1}, k_{2}, k_{3}\right)} .
$$

The functions $\mathbf{f}_{0}$ form a $p$-adic family. The evaluation at triple weight $k=$ $\left(k_{1}, k_{2}, k_{3}\right)$ of the $p$-adic coordinate with respect to $p$-adic family $\mathbf{f}_{0}$ is expressed through the triple Petersson scalar product with $\mathbf{f}^{0}$, which is algebraically orthogonal to $\mathbf{f}_{0}$ in the sense of Hida [Hi90] for classical weights (and in [PaTV] for Coleman's families).

We prove that $U$ is a completely continuos $\mathcal{A}$-linear operator on a certain Coleman's submodule $\mathcal{M}(\mathcal{A})^{\dagger}$ of Serre's type module $\mathcal{M}(\mathcal{A})$. Then the projection $\pi_{\lambda}$ exists (on this submodule) due to general results of Serre and Coleman, see $\mathrm{CoPB}$, SePB].

We show that there exists an element $\tilde{\mathcal{E}}(-r, \chi) \in \mathcal{M}(\mathcal{A})^{\dagger}$ such that at each weight $\left(k_{1}, k_{2}, k_{3}\right)$ the equality holds: $\left\langle\mathbf{f}^{0}, \mathcal{E}(-r, \chi)\right\rangle=\left\langle\mathbf{f}^{0}, \pi_{\lambda}(\tilde{\mathcal{E}}(-r, \chi))\right\rangle$, and the product can be expressed through certain coefficients the series $\tilde{\mathcal{E}}(-r, \chi)$ which are the same as those of $\mathcal{E}(-r, \chi)$.

- Key point: modular admissible measures. Let us write for simplicity: $\mathcal{E}(-r, \chi)$ for $\tilde{\mathcal{E}}(-r, \chi)$

$$
\mathcal{M}_{T}(\mathcal{A}) \text { instead of } \mathcal{M}_{T}(\mathcal{A})^{\dagger} \text { (Coleman's submodule) }
$$

One defines admissible $p$-adic measures $\tilde{\Phi}^{\lambda}$ with values in Banach $\mathcal{A}$-modules $\mathcal{M}_{T}^{\lambda}(\mathcal{A})$ which are locally free of finite rank, using the test functions: $\int_{Y} \chi y_{p}^{r} \tilde{\Phi}^{\lambda}=$ $\pi_{\lambda}(\mathcal{E}(-r, \chi))$.

Consider the evaluation maps $e v_{\mathbf{s}}: \mathcal{A} \rightarrow \mathbb{C}_{p}$ for any $p$-adic triple weights $\mathbf{s}=$ $\left(s_{1}, s_{2}, s_{3}\right) \in \mathcal{B}$. 
- Passage from values in modular forms to scalar values: apply an algebraic $\mathcal{A}$-linear form $\mathcal{M}_{T}^{\lambda}(\mathcal{A}) \stackrel{\ell_{T}}{\rightarrow} \mathcal{A}$ to the constructed measure $\tilde{\Phi}^{\lambda}$ (in modular forms), and the evaluation maps $\mathcal{A} \stackrel{e v_{\mathrm{s}}}{\longrightarrow} \mathbb{C}_{p}$ for any $p$-adic triple weights $\mathbf{s} \in X^{3}$.

The linear form $\ell_{T}$ is an algebraic version of the Petersson product (a geometric meaning of $\ell_{T}$ : the first coordinate in an (orthogonal) $\mathcal{A}$-basis of eigenfunctions of all Hecke operators $T_{q}$ for $q \nmid N p$, with the first basis element $\mathbf{f}_{0} \in \mathcal{M}^{\lambda}(\mathcal{A})$ ).

Using the evaluation map and the Mellin transform. We obtain the measure $\mu=\ell_{T}\left(\tilde{\Phi}^{\lambda}\right)$ with values in $\mathcal{A}$ on the profinite group $Y$.

- Construct an analytic function $\mathcal{L}_{\mu}: X \rightarrow \mathcal{A}=\mathcal{A}(\mathcal{B})$ as the $p$-adic Mellin transform $\mathcal{L}_{\mu}(x)=\int_{Y} x(y) d \mu(y) \in \mathcal{A}, x \in X$.

- Solution: the function in question $\mathcal{L}_{\mu}(x, \mathbf{s})$ is given by evaluation of $\mathcal{L}_{\mu}(x)$ at $\mathbf{s}=\left(s_{1}, s_{2}, s_{3}\right) \in \mathcal{B}$ : this is a $p$-adic analytic function in four variables

$$
\begin{array}{r}
(x, \mathbf{s}) \in X \times \mathcal{B}_{1} \times \mathcal{B}_{2} \times \mathcal{B}_{3} \subset X \times X \times X \times X \\
\mathcal{L}_{\mu}(x, \mathbf{s}):=e v_{\mathbf{s}}\left(\mathcal{L}_{\mu}(x)\right) \quad\left(x \in X, \mathbf{s} \in \mathcal{B}_{1} \times \mathcal{B}_{2} \times \mathcal{B}_{3}, \quad \mathcal{L}_{\mu}(x) \in \mathcal{A}\right) .
\end{array}
$$

Final step: comparison between $\mathbb{C}$ and $\mathbb{C}_{p}$. $\bullet$ We check an equality relating the values of the constructed analytic function $\mathcal{L}_{\mu}(x, \mathbf{s})$ at the arithmetical characters $x=y_{p}^{r} \chi \in X$, and at triple weights $\mathbf{s}=\left(k_{1}, k_{2}, k_{3}\right) \in \mathcal{B}$, with the normalized critical special values

$$
L^{*}\left(f_{1, k_{1}} \otimes f_{2, k_{2}} \otimes f_{3, k_{3}}, k_{2}+k_{3}-2-r, \chi\right) \quad\left(r=0, \cdots, k_{2}+k_{3}-k_{1}-2\right),
$$

for certain Dirichlet characters $\chi \bmod N p^{v}, v \geq 1$. These are algebraic numbers, embedded into $\mathbb{C}_{p}=\widehat{\overline{\mathbb{Q}}}_{p}$ (the Tate field of $p$-adic numbers). The normalisation of $L^{*}$ includes at the same time Gauss sums, Petersson scalar products, powers of $\pi$, the product $\lambda_{p}\left(k_{1}, k_{2}, k_{3}\right)$, and a certain finite Euler product.

We refer to Theorem 0.3 at p.556 of [PaTV], where the explicit form of such finite Euler product is given in the case of the $L$-function of one Coleman's family.

\section{Arithmetical NeARly holomorphic modular Forms}

Arithmetical nearly holomorphic modular forms (the elliptic case). Let $\mathcal{A}$ be a commutative ring (a subring of $\mathbb{C}$ or $\mathbb{C}_{p}$ )

Arithmetical nearly holomorphic modular forms (in the sense of Shimura, ShiAr] are certain formal series

$$
g=\sum_{n=0}^{\infty} a(n ; R) q^{n} \in \mathcal{A} \llbracket q \rrbracket[R], \text { with the property }
$$


that for $\mathcal{A}=\mathbb{C}, z=x+i y \in \mathbb{H}, R=(4 \pi y)^{-1}$, the series converges to a $\mathrm{C}^{\infty}$ modular form on $\mathbb{H}$ of a given weight $k$ and Dirichlet character $\psi$. The coefficients $a(n ; R)$ are polynomials in $\mathcal{A}[R]$. If $\operatorname{deg}_{R} a(n ; R) \leq r$ for all $n$, we call $g$ nearly holomorphic of type $r$ (it is annihilated by $\left(\frac{\partial}{\partial \bar{z}}\right)^{r+1}$, see [ShiAr]).

. We use the notation $\mathcal{M}_{k, r}(N, \psi, \mathcal{A})$ or $\tilde{\mathcal{M}}(N, \psi, \mathcal{A})$ for $\mathcal{A}$-modules of such forms (In our constructions the weight $k$ varies).

A known example (see the introduction to [ShiAr]) is given by the series

$$
\begin{aligned}
& -12 R+E_{2}:=-12 R+1-24 \sum_{n=1}^{\infty} \sigma_{1}(n) q^{n} \\
& =\frac{3}{\pi^{2}} \lim _{s \rightarrow 0} y^{s} \sum_{m_{1}, m_{2} \in \mathbb{Z}}^{\prime}\left(m_{1}+m_{2} z\right)^{-2}\left|m_{1}+m_{2} z\right|^{-2 s},\left(R=(4 \pi y)^{-1}\right)
\end{aligned}
$$

where $\sigma_{1}(n)=\sum_{d \mid n} d$.

The action of the Shimura differential operator

$$
\delta_{k}: \mathcal{M}_{k, r}(N, \psi, \mathcal{A}) \rightarrow \mathcal{M}_{k+2, r+1}(N, \psi, \mathcal{A}),
$$

is given over $\mathbb{C}$ by $\delta_{k}(f)=\left(\frac{1}{2 \pi i} \frac{\partial}{\partial z}-\frac{k}{4 \pi y}\right) f$.

This operator is a correction of the Ramanujan operator

$$
\theta\left(\sum_{n=0}^{\infty} a_{n} q^{n}\right)=\sum_{n=1}^{\infty} n a_{n} q^{n}=\frac{1}{2 \pi i} \frac{\partial}{\partial z}\left(\sum_{n=0}^{\infty} a_{n} q^{n}\right)=q \frac{\partial}{\partial q}\left(\sum_{n=0}^{\infty} a_{n} q^{n}\right),
$$

which does not preserve the modularity. For example $\theta \Delta=E_{2} \Delta$, where $E_{2}$ is a quasimodular form (in the sense of Kaneko and Zagier, see [Ka-Za]).

Notice that $\delta_{k} f=(\theta-k R) f$, and that Serre's operator $f \mapsto \theta f-\frac{k}{12} E_{2} f$ takes $\mathcal{M}_{k}$ to $\mathcal{M}_{k+2}$.

Note that that the arithmetical twist operator

$$
\theta_{\chi}\left(\sum_{n=0}^{\infty} a_{n} q^{n}\right)=\sum_{n=1}^{\infty} \chi(n) a_{n} q^{n}
$$

is a natural analog of the Ramanujan operator.

Triple arithmetical modular forms. Let $\mathcal{A}$ be a commutative ring. The tensor product over $\mathcal{A}$

$$
\mathcal{M}_{\mathbf{k}, r, T}(N, \psi, \mathcal{A}):=\mathcal{M}_{k_{1}, r}\left(N, \psi_{1}, \mathcal{A}\right) \otimes \mathcal{M}_{k_{2}, r}\left(N, \psi_{2}, \mathcal{A}\right) \otimes \mathcal{M}_{k_{3}, r}\left(N, \psi_{3}, \mathcal{A}\right)
$$


consists of triple arithmetical modular forms as certain formal series of the form

$$
\begin{aligned}
& g=\sum_{n_{1}, n_{2}, n_{3}=0}^{\infty} a\left(n_{1}, n_{2}, n_{3} ; R_{1}, R_{2}, R_{3}\right) q_{1}^{n_{1}} q_{2}^{n_{2}} q_{3}^{n_{3}} \\
& \in \mathcal{A} \llbracket q_{1}, q_{2}, q_{3} \rrbracket\left[R_{1}, R_{2}, R_{3}\right], \text { where } z_{j}=x_{j}+i y_{j} \in \mathbb{H}, R_{j}=\left(4 \pi y_{j}\right)^{-1},
\end{aligned}
$$

with the property that for $\mathcal{A}=\mathbb{C}$, the series converges to a $\mathfrak{C}^{\infty}$-modular form on $\mathbb{H}^{3}$ of a given weight $\left(k_{1}, k_{2}, k_{3}\right)$ and character $\left(\psi_{1}, \psi_{2}, \psi_{3}\right), j=1,2,3$. The coefficients $a\left(n_{1}, n_{2}, n_{3} ; R_{1}, R_{2}, R_{3}\right)$ are polynomials in $\mathcal{A}\left[R_{1}, R_{2}, R_{3}\right]$. Examples of such modular forms come from the restriction to the diagonal of Siegel modular forms of degree 3 .

\section{Siegel-Eisenstein SeRIES}

Siegel modular groups. Let $J_{2 m}=\left(\begin{array}{cc}0_{m} & -1_{m} \\ 1_{m} & 0_{m}\end{array}\right)$. The symplectic group

$$
\mathrm{Sp}_{m}(\mathbb{R})=\left\{\left.g \in \mathrm{GL}_{2 m}(\mathbb{R})\right|^{t} g \cdot J_{2 m} g=J_{2 m}\right\},
$$

acts on the Siegel upper half plane

$$
\mathbb{H}_{m}=\left\{z={ }^{t} z \in M_{m}(\mathbb{C}) \mid \operatorname{Im} z>0\right\}
$$

by $g(z)=(a z+b)(c z+d)^{-1}$, where we use the bloc notation $g=\left(\begin{array}{ll}a & b \\ c & d\end{array}\right) \in \operatorname{Sp}_{2 m}(\mathbb{R})$. We use the congruence subgroup $\Gamma_{0}^{m}(N)=\left\{\gamma \in \operatorname{Sp}_{m}(\mathbb{Z}) \mid \gamma \equiv\left(\begin{array}{l}* * \\ 0 *\end{array}\right) \bmod N\right\} \subset$ $\mathrm{Sp}_{m}(\mathbb{Z})$.

A Siegel modular form. $f \in \mathcal{M}_{k}\left(\Gamma_{0}^{m}(N), \chi\right)$ of degree $m>1$, weight $k$ and a Dirichlet chracter $\chi \bmod N$ is a holomorphic function $f: \mathbb{H}_{m} \rightarrow \mathbb{C}$ such that for every $\gamma=\left(\begin{array}{ll}a & b \\ c & d\end{array}\right) \in \Gamma_{0}^{m}(N)$ one has

$$
f(\gamma(z))=\chi(\operatorname{det} d) \operatorname{det}(c z+d)^{k} f(z) .
$$

The Fourier expansion of $f$ uses the symbol

$$
q^{\mathcal{T}}=\exp (2 \pi i \operatorname{tr}(\mathcal{T} z))=\prod_{i=1}^{m} q_{i i}^{\mathcal{T}_{i i}} \prod_{i<j} q_{i j}^{2 \mathcal{T}_{i j}} \in \mathbb{C}\left[q_{11}, \cdots, q_{m m}, q_{i j}, q_{i j}^{-1}\right]_{1 \leq i<j \leq m}
$$

for each Fourier coefficient, where $q_{i j}=\exp \left(2 \pi\left(\sqrt{-1} z_{i, j}\right)\right.$, and $\mathcal{T}$ in the semi-group $B_{m}=\left\{\mathcal{T}={ }^{t} \mathcal{T} \geq 0 \mid \mathcal{T}\right.$ half-integral $\}$. We may consider $f(z)=\sum_{\mathcal{T} \in B_{m}} a(\mathcal{T}) q^{\mathcal{T}} \in$ $\mathbb{C} \llbracket q^{B_{m}} \rrbracket$ as a formal $q$-expansion in $\mathbb{C} \llbracket q^{B_{m}} \rrbracket$ (the subring of $\left.\mathbb{C} \llbracket q_{11}, \ldots, q_{m m} \rrbracket\left[q_{i j}, q_{i j}^{-1}\right]\right)$ generated by all $q^{\mathcal{T}}$ ). 
Siegel-Eisenstein series.

EXAMPLE 5.1 ( Nag2, p.408).

$$
\begin{aligned}
E_{4}^{(2)}(z)= & 1+240 q_{11}+240 q_{22}+2160 q_{11}^{2}+\left(240 q_{12}^{-2}+13440 q_{12}^{-1}\right. \\
& \left.+30240+13440 q_{12}+240 q_{12}^{2}\right) q_{11} q_{22}+2160 q_{22}^{2}+\ldots \\
E_{6}^{(2)}(z)= & 1-504 q_{11}-504 q_{22}-16632 q_{11}^{2}+\left(-540 q_{12}^{-2}+44352 q_{12}^{-1}\right. \\
& \left.+166320+44352 q_{12}-504 q_{12}^{2}\right) q_{11} q_{22}-16632 q_{22}^{2}+\ldots
\end{aligned}
$$

\section{Arithmetical nearly holomorphic Siegel modular forms.}

Arithmetical Siegel modular forms. Consider a commutative ring $\mathcal{A}$, the formal variables $q=\left(q_{i, j}\right)_{i, j=1, \ldots, m}, R=\left(R_{i, j}\right)_{i, j=1, \ldots, m}$, and the ring of formal Fourier series

$$
\mathcal{A} \llbracket q^{B_{m}} \rrbracket\left[R_{i, j}\right]=\left\{f=\sum_{\mathcal{T} \in B_{m}} a(\mathcal{T}, R) q^{\mathcal{T}} \mid a(\mathcal{T}, R) \in \mathcal{A}\left[R_{i, j}\right]\right\}
$$

(over the complex numbers this notation corresponds to $q^{\mathcal{T}}=\exp (2 \pi i \operatorname{tr}(\mathcal{T} z))$, $\left.R=(4 \pi \operatorname{Im}(z))^{-1}\right)$.

The formal Fourier expansion of a nearly holomorphic Siegel modular form $f$ with coefficients in $\mathcal{A}$ is a certain element of $\mathcal{A} \llbracket q^{B_{m}} \rrbracket\left[R_{i, j}\right]$. We call $f$ arithmetical in the sense of Shimura [ShiAr], if $\mathcal{A}=\overline{\mathbb{Q}}$.

\subsection{Algebraic differential operators of Maass and Shimura.}

Maass differential operator. Let us consider the Maass differential operator (see Maa]) $\Delta_{m}$ of degree $m$, acting on complex $\mathcal{C}^{\infty}$-functions on $\mathbb{H}_{m}$ by:

$$
\Delta_{m}=\operatorname{det}\left(\tilde{\partial}_{i j}\right), \quad \tilde{\partial}_{i j}=2^{-1}\left(1+\delta_{i j}\right) \partial / \partial_{i j},
$$

its algebraic version is the Ramanujan operator of degree $m$ :

$$
\Theta_{m}:=\operatorname{det}\left(\frac{1}{2 \pi i} \tilde{\partial}_{i j}\right)=\operatorname{det}\left(\theta_{i j}\right)=\frac{1}{(2 \pi i)^{m}} \Delta_{m},
$$

where $\Theta_{m}\left(q^{\mathcal{T}}\right)=\operatorname{det}(\mathcal{T}) q^{\mathcal{T}}$.

Shimura differential operator. The Shimura differential operator (see [Shi76, ShiAr]):

$$
\delta_{k} f(z)=\operatorname{det}(R)^{k+1-\varkappa} \Theta_{m}\left[\operatorname{det}(R)^{\varkappa-1-k} f\right] \text {, where } R=(4 \pi y)^{-1},
$$


acts on arithmetic nearly holomorphic Siegel modular forms, and the composition is defined

$$
\delta_{k}^{(r)}=\delta_{k+2 r-2} \circ \cdots \circ \delta_{k}: \tilde{\mathcal{M}}_{k}^{m}(N, \psi ; \overline{\mathbb{Q}}) \rightarrow \tilde{\mathcal{M}}_{k+2 r m}^{m}(N, \psi ; \overline{\mathbb{Q}}),
$$

where

$$
\delta_{k} f(z)=\left(\frac{-1}{4 \pi}\right)^{m} \operatorname{det}(y)^{-1} \operatorname{det}(z-\bar{z})^{\varkappa-k} \Delta_{m}\left[\operatorname{det}(z-\bar{z})^{k-\varkappa+1} f(z)\right] .
$$

Universal polynomials $Q(R, \mathcal{T} ; k, r)$. Let $f=\sum_{\mathcal{T} \in B_{m}} c(\mathcal{T}) q^{\mathcal{T}} \in \mathcal{M}_{k}^{m}(N, \psi)$ be a formal holomorphic Fourier expansion. One shows that $\delta_{k}^{(r)} f$ is given by

$$
\delta_{k}^{(r)} f=\sum_{\mathcal{T} \in B_{m}} Q(R, \mathcal{T} ; k, r) c(\mathcal{T}) q^{\mathcal{T}}
$$

Here we use a universal polynomial (5.10) which can be defined for all $k \in \mathbb{C}$, and it expresses the action of the Shimura operator on the exponential (of degree $m)$ :

$$
\delta_{k}^{(r)}\left(q^{\mathcal{T}}\right)=Q(R, \mathcal{T} ; k, r) q^{\mathcal{T}}
$$

If $m=1, r$ arbitrary (see [Shi76]), $\delta_{k}^{(r)}=\sum_{j=0}^{r}(-1)^{r-j}\left(\begin{array}{l}r \\ j\end{array}\right) \frac{\Gamma(k+r)}{\Gamma(k+j)} R^{r-j} \theta^{j}$, $Q(R, n ; k, r)=\sum_{j=0}^{r}(-1)^{r-j}\left(\begin{array}{l}r \\ j\end{array}\right) \frac{\Gamma(k+r)}{\Gamma(k+j)} R^{r-j} n^{j}$.

If $r=1, m$ arbitrary, one has (see [Maa]):

$$
\delta_{k} f(z)=\sum_{\mathcal{T} \in B_{m}} c(\mathcal{T}) \sum_{l=0}^{m}(-1)^{m-l} c_{m-l}(k+1-\varkappa) \operatorname{tr}\left({ }^{t} \rho_{m-l}(R) \cdot \rho_{l}^{\star}(\mathcal{T})\right) q^{\mathcal{T}}
$$

where $R=(4 \pi y)^{-1}=\left(R_{i, j}\right) \in \mathrm{M}_{m}(\mathbb{R}), c_{m}(\alpha)=\frac{\Gamma_{m}(\alpha+\kappa)}{\Gamma_{m}(\alpha+\kappa-1)}, \quad \Gamma_{m}(s)=$ $\pi^{m(m-1) / 4} \prod_{j=0}^{m-1} \Gamma(s-(j / 2))$.

Here we use the natural representation $\rho_{r}: \mathrm{GL}_{m}(\mathbb{C}) \longrightarrow \mathrm{GL}\left(\wedge^{r} \mathbb{C}^{m}\right)(0 \leq$ $r \leq m$ ) of the group $\mathrm{GL}_{m}(\mathbb{C})$ on the vector space $\Lambda^{r} \mathbb{C}^{m}$. Thus $\rho_{r}(z)$ is a matrix of size $\left(\begin{array}{c}m \\ r\end{array}\right) \times\left(\begin{array}{c}m \\ r\end{array}\right)$ composed of the subdeterminants of $z$ of degree $r$. Put $\rho_{r}^{\star}(z)=\operatorname{det}(z) \rho_{m-r}\left({ }^{t} z\right)^{-1}$.

Then the representations $\rho_{r}$ and $\rho_{r}^{\star}$ turn out to be polynomial representations. 
. In general (see CourPa, Theorem 3.14) one has:

$$
\begin{aligned}
Q(R, \mathcal{T}) & =Q(R, \mathcal{T} ; k, r) \\
& =\sum_{t=0}^{r}\left(\begin{array}{l}
r \\
t
\end{array}\right) \operatorname{det}(\mathcal{T})^{r-t} \sum_{|L| \leq m t-t} R_{L}(\kappa-k-r) Q_{L}(R, \mathcal{T}), \\
Q_{L}(R, \mathcal{T}) & \left.=\operatorname{tr}\left({ }^{t} \rho_{m-l_{1}}(R) \rho_{l_{1}}^{\star}(\mathcal{T})\right) \cdot \ldots \cdot \operatorname{tr}\left({ }^{t} \rho_{m-l_{t}}(R) \rho_{l_{t}}^{\star}(\mathcal{T})\right)\right) .
\end{aligned}
$$

In 5.10$), L$ goes over all the multi-indices $0 \leq l_{1} \leq \cdots \leq l_{t} \leq m$, such that $|L|=l_{1}+\cdots+l_{t} \leq m t-t$, and $R_{L}(\beta) \in \mathbb{Z}[1 / 2][\beta]$ in (5.10) are polynomials in $\beta$ of degree $(m t-|L|)$ (used with $\beta=\kappa-k-r$ ).

Note the differentiation rule of degree $m$ (see [Sh83, p.466):

$\Delta(f g)=\sum_{r=0}^{m} \operatorname{tr}\left({ }^{t} \rho_{r}(\tilde{\partial} / \partial z) f \cdot \rho_{m-r}^{\star}(\tilde{\partial} / \partial z) g\right)$. As in 5.8p, we write here $\tilde{\partial} / \partial z=$ $\left(\tilde{\partial} / \partial z_{i j}\right)$ for the matrix with entries $\tilde{\partial}_{i j}=2^{-1}\left(1+\delta_{i j}\right) \partial / \partial_{i j}$.

EXAmple 5.2 (Siegel-Eisenstein series of odd degree and higher level).

$$
\begin{aligned}
& G^{*}(z, s ; k, \boldsymbol{\psi}, N) \\
& =\operatorname{det}(y)^{s} \sum_{c, d} \boldsymbol{\psi}(\operatorname{det} c) \operatorname{det}(c z+d)^{-k}|\operatorname{det}(c z+d)|^{-2 s} . \\
& \cdot \tilde{\Gamma}(k, s) L_{N}(k+2 s, \boldsymbol{\psi})\left(\prod_{i=1}^{[m / 2]} L_{N}\left(2 k+4 s-2 i, \boldsymbol{\psi}^{2}\right)\right) \text {, where }
\end{aligned}
$$

$(c, d)$ runs over all "non-associated coprime symmetric pairs" with $\operatorname{det}(c)$ coprime to $N, \kappa=(m+1) / 2$, and for $m$ odd the $\Gamma$-factor has the form:

$\tilde{\Gamma}(k, s)=i^{m k} 2^{-m(k+1)} \pi^{-m(s+k)} \Gamma_{m}(k+s)$.

We use this series with $\boldsymbol{\psi}=\chi^{2} \psi_{1} \psi_{2} \bar{\psi}_{3}, k=k_{2}+k_{3}-k_{1} \geq 2, m=3, \kappa=$ $\frac{m+1}{2}=2,[m / 2]=1$.

Theorem 5.3 (Siegel, Shimura [Sh83], P. Feit [Fei86]). Let $m$ be an odd integer such that $2 k>m$, and $N>1$ be an integer, then:

For an integer $s$ such that $s=-r, 0 \leq r \leq k-\kappa$, there is the following Fourier expansion

$$
G^{\star}(z,-r)=G^{\star}(z,-r ; k, \boldsymbol{\psi}, N)=\sum_{A_{m} \ni \mathcal{T} \geq 0} a(\mathcal{T}, R) q^{\mathcal{T}}
$$


where for $s>(m+2-2 k) / 4$ in (5.12) the only non-zero terms occur for positive definite $\mathcal{T}>0$,

$$
\begin{aligned}
& a(\mathcal{T}, R)=M(\mathcal{T}, \boldsymbol{\psi}, k-2 r) \cdot \operatorname{det}(\mathcal{T})^{k-2 r-\kappa} Q(R, \mathcal{T} ; k-2 r, r), \\
& M(\mathcal{T}, k-2 r, \boldsymbol{\psi})=\prod_{\ell \mid \operatorname{det}(2 \mathcal{T})} M_{\ell}\left(\mathcal{T}, \boldsymbol{\psi}(\ell) \ell^{-k+2 r}\right)
\end{aligned}
$$

polynomials $Q(R, \mathcal{T} ; k-2 r, r)$ are given by (5.10), and for all $\mathcal{T}>0, \mathcal{T} \in A_{m}$, is a finite Euler product, in which $M_{\ell}(\mathcal{T}, x) \in \mathbb{Z}[x]$.

\section{Statement of the Main Result}

Main Theorem (on p-adic analytic function in four variables). The following Main Theorem corresponds to Theorem 0.3 at p.556 of [PaTV] in the situation of the $L$-function attached to one Coleman's family.

Main Theorem 6.1. 1) The function $\mathcal{L}_{\boldsymbol{f}}:\left(s, k_{1}, k_{2}, k_{3}\right) \mapsto \frac{\left\langle\boldsymbol{f}^{0}, \mathcal{E}(-r, \chi)\right\rangle}{\left\langle\boldsymbol{f}^{0}, \boldsymbol{f}_{0}\right\rangle}$ extends to a $p$-adic analytic function on four variables $\left(\chi \cdot y_{p}^{r}, k_{1}, k_{2}, k_{3}\right) \in X \times \mathcal{B}_{1} \times \mathcal{B}_{2} \times \mathcal{B}_{3}$;

2) Comparison of complex and p-adic values: for all $\left(k_{1}, k_{2}, k_{3}\right)$ in an affinoid neighborhood $\mathcal{B}=\mathcal{B}_{1} \times \mathcal{B}_{2} \times \mathcal{B}_{3} \subset X^{3}$, satisfying $k_{1} \leq k_{2}+k_{3}-2$ : the values at $s=k_{2}+k_{3}-2-r$ coincide with the normalized critical special values

$$
\begin{aligned}
& L^{*}\left(f_{1, k_{1}} \otimes f_{2, k_{2}} \otimes f_{3, k_{3}}, k_{2}+k_{3}-2-r, \chi\right) \\
& \left(r=0, \cdots, k_{2}+k_{3}-k_{1}-2\right),
\end{aligned}
$$

for Dirichlet characters $\chi \bmod N p^{v}, v \geq 1$, such that all three corresponding Dirichlet characters $\chi_{j}$ have $N p$-complete conductors:

$$
\begin{aligned}
& \chi_{1} \bmod N p^{v}=\chi, \chi_{2} \bmod N p^{v}=\psi_{2} \bar{\psi}_{3} \chi, \\
& \chi_{3} \bmod N p^{v}=\psi_{1} \bar{\psi}_{3} \chi, \boldsymbol{\psi}=\chi^{2} \psi_{1} \psi_{2} \bar{\psi}_{3} .
\end{aligned}
$$

The normalisation of $L^{*}$ in 6.15) is the same as in Theorem $C$ below.

3) Dependence on $x \in X$ : let $H=\left[\operatorname{2ord}_{p}(\lambda)\right]+1$. For any fixed $\left(k_{1}, k_{2}, k_{3}\right) \in \mathcal{B}$ and $x=\chi \cdot y_{p}^{r}$ the function

$$
x \longmapsto \frac{\left\langle f^{0}, \mathcal{E}(-r, \chi)\right\rangle}{\left\langle f^{0}, f_{0}\right\rangle}
$$

extends to a $p$-adic analytic function of type $o\left(\log ^{H}(\cdot)\right)$ of the variable $x \in X$. 
REMARK. The function $\mathcal{L}_{\mathbf{f}}$ depends on the variables $\left(s, k_{1}, k_{2}, k_{3}\right)$ in a different way: it is a mixture of the $p$-adic Mellin transform (in $s$ ), and of a rigid analytic function (in $k_{1}, k_{2}, k_{3}$ ).

Outline of the proof. The proof follows the lines given in Sections 5-7 in [PaTV] (the case of the $L$-function of one Coleman's family).

1) - (compare with Section 5 in [PaTV] $)$. At each classical weight $\left(k_{1}, k_{2}, k_{3}\right)$ let us use the equality

$$
\left\langle\mathbf{f}^{0}, \mathcal{E}(-r, \chi)\right\rangle=\left\langle\mathbf{f}^{0}, \pi_{\lambda}(\mathcal{E}(-r, \chi))\right\rangle
$$

which is deduced from the definition of the projector $\pi_{\lambda}$ : $\operatorname{Ker} \pi_{\lambda}:=\bigcap_{n \geq 1} \operatorname{Im}\left(U_{T}-\right.$ $\lambda I)^{n}, \operatorname{Im} \pi_{\lambda}:=\bigcup_{n \geq 1} \operatorname{Ker}\left(U_{T}-\lambda I\right)^{n}$.

Notice that the coefficients of $\mathcal{E}(-r, \chi) \in \mathcal{N}(\mathcal{A})$ depend $p$-adic analytically on $\left(k_{1}, k_{2}, k_{3}\right) \in \mathcal{B}=\mathcal{B}_{1} \times \mathcal{B}_{2} \times \mathcal{B}_{3}$, where $\mathcal{A}=\mathcal{A}\left(\mathcal{B}_{1} \times \mathcal{B}_{2} \times \mathcal{B}_{3}\right)$ is the $p$-adic Banach algebra of rigid-analytic functions on $\mathcal{B}$.

Interpolation to all $p$-adic weights: - At each classical weight $\left(k_{1}, k_{2}, k_{3}\right)$ the scalar product $\left\langle\mathbf{f}^{0}, \mathcal{E}(-r, \chi)\right\rangle$ is given by the first coordinate of $\pi_{\lambda}(\mathcal{E}(-r, \chi))$ with respect to an orthogonal basis of $\mathcal{M}^{\lambda}(\mathcal{A})$ containing $\mathbf{f}_{0}$ with respect to Hida's algebraic Petersson product $\langle g, h\rangle_{a}:=\left\langle g^{\rho} \mid\left(\begin{array}{cc}0 & -1 \\ N p & 0\end{array}\right), h\right\rangle$, see [Hi90].

Let us extend the linear form $\ell(h)=\frac{\left\langle\mathbf{f}^{0}, h\right\rangle}{\left\langle\mathbf{f}^{0}, \mathbf{f}_{0}\right\rangle}$ (defined firs only for classical weights), to Coleman's type submodule of overconvergent families $h \in \mathcal{M}^{\lambda}(\mathcal{A})^{\dagger} \subset$ $\mathcal{M}^{\lambda}(\mathcal{A})$ as the first coordinate of $h$ with respect to some $\mathcal{A}$-basis of eigenfunctions of all (triple) Hecke operators $T_{q}$ for $q \nmid N p$, having the first basis vector $\mathbf{f}_{0} \in$ $\mathcal{M}^{\lambda}(\mathcal{A})^{\dagger}$.

The linear form $\ell$ can be characterized as a normalized eigenfunction of the adjoint Atkin's operator, acting on the dual $\mathcal{A}$-module of $\mathcal{M}^{\lambda}(\mathcal{A})^{\dagger}: \ell\left(\mathbf{f}_{0}\right)=1$.

In order to extend $\ell$ to $h=\mathcal{E}(-r, \chi)$, we need to choose a certain representative of $\mathcal{E}(-r, \chi)$ in the $\mathcal{A}$-submodule $\mathcal{M}^{\lambda}(\mathcal{A})^{\dagger}$, which is locally free of finite rank.

A representative of $\mathcal{E}(-r, \chi)$ in the (locally free of finite rank $\mathcal{A}$-submodule) $\mathcal{M}^{\lambda}(\mathcal{A})^{\dagger}$. (compare with Section 6 in [PaTV] in the case of the $L$-function of one Coleman's family). Choose a (local) basis $\ell^{1}, \cdots, \ell^{n}$ given by some triple Fourier coefficients of the dual (locally free of finite rank) $\mathcal{A}$-module $\mathcal{M}^{\lambda}(\mathcal{A})^{\dagger *}$.

Then define

$$
\ell=\beta_{1} \ell^{1}+\cdots+\beta_{n} \ell^{n}
$$

where $\beta_{i}=\ell\left(\ell_{i}\right) \in \mathcal{A}$, and $\ell_{i}$ denotes the dual basis of $\mathcal{M}^{\lambda}(\mathcal{A})^{\dagger}: \ell^{j}\left(\ell_{i}\right)=\delta_{i j}$. 
At each $p$-adic weight $\left(k_{1}, k_{2}, k_{3}\right) \in \mathcal{B}$ let us define

$$
\left.\ell(\mathcal{E}(-r, \chi)):=\beta_{1} \ell^{1}(\mathcal{E}(-r, \chi))+\cdots+\beta_{n} \ell^{n}(\mathcal{E}(-r, \chi)) \text { (belongs to } \mathcal{A}\right),
$$

where $\beta_{i}=\ell\left(\ell_{i}\right) \in \mathcal{A}$, and $\ell^{i}(\mathcal{E}(-r, \chi)) \in \mathcal{A}$ are certain Fourier coefficients of the seies $\mathcal{E}(-r, \chi)$.

Conclusion. There exists an element $\tilde{\mathcal{E}}(-r, \chi) \in \mathcal{M}^{\lambda}(\mathcal{A})^{\dagger} \subset \mathcal{M}(\mathcal{A})^{\dagger}$ such that

$$
\ell(\mathcal{E}(-r, \chi))=\ell(\tilde{\mathcal{E}}(-r, \chi))
$$

(at each triple weight $\left.\left(k_{1}, k_{2}, k_{3}\right)\right)$. In fact, let us define

$$
\begin{aligned}
& \quad \tilde{\mathcal{E}}(-r, \chi):=\ell^{1}(\mathcal{E}(-r, \chi)) \ell_{1}+\cdots+\ell^{n}(\mathcal{E}(-r, \chi)) \ell_{n} \\
& \Rightarrow \ell(\tilde{\mathcal{E}}(-r, \chi))=\ell\left(\ell_{1}\right) \ell^{1}(\mathcal{E}(-r, \chi))+\cdots+\ell\left(\ell_{n}\right) \ell^{n}(\mathcal{E}(-r, \chi)) \\
& =\beta_{1} \ell^{1}(\mathcal{E}(-r, \chi))+\cdots+\beta_{n} \ell^{n}(\mathcal{E}(-r, \chi)) \\
& \left.=\ell(\mathcal{E}(-r, \chi)) \text { (at each weight }\left(k_{1}, k_{2}, k_{3}\right)\right) .
\end{aligned}
$$

Thus, the dependence of $\ell(\mathcal{E}(-r, \chi)) \in \mathcal{A}$ on $\left(k_{1}, k_{2}, k_{3}\right) \in X^{3}$ is $p$-adic analytic.

In order to prove the remaining statements 2), 3), the dependence on $x=\chi \cdot y_{p}^{r}$ is studied in the next section.

\section{Distributions and admissible measures}

Distributions and measures with values in Banach modules. We refer to Section 4 of [PaTV] for similar constructions in the case of the $L$-function of one Coleman's family.

$\begin{array}{lll} & \mathcal{A} & \text { (a } p \text {-adic Banach algebra) } \\ \text { Notation. } & V & \text { (an } \mathcal{A} \text {-module) } \\ & \mathcal{C}(Y, \mathcal{A}) & \text { (the } \mathcal{A} \text {-Banach algebra } \\ & \cup & \text { of continuous functions on } Y \text { ) } \\ \mathrm{e}^{\text {loc-const }}(Y, \mathcal{A}) & \begin{array}{l}\text { (the } \mathcal{A} \text {-algebra } \\ \text { of locally constant functions on } Y \text { ) }\end{array}\end{array}$

Definition 7.1 (Distributions and measures). a) $A$ distribution $\mathcal{D}$ on $Y$ with values in $V$ is an $\mathcal{A}$-linear form

$$
\mathcal{D}: \mathrm{e}^{\text {loc-const }}(Y, \mathcal{A}) \rightarrow V, \quad \varphi \mapsto \mathcal{D}(\varphi)=\int_{Y} \varphi d \mathcal{D} .
$$

b) $A$ measure $\mu$ on $Y$ with values in $V$ is a continuous $\mathcal{A}$-linear form

$$
\mu: \mathcal{C}(Y, \mathcal{A}) \rightarrow V, \quad \varphi \mapsto \int_{Y} \varphi d \mu
$$


The integral $\int_{Y} \varphi d \mu$ can be defined for any continuous function $\varphi$, and any bounded distribution $\mu$, using the Riemann sums.

\section{Admissible measures of Amice-Vélu.}

Admissible measures. Let $h$ be a positive integer. A more delicate notion of an $h$-admissible measure was introduced in $\mathrm{Am}-\mathrm{V}$ ] by Y. Amice, J. Vélu (see also [MTT, [V]):

DEFINITION 7.2.

a) For $h \in \mathbb{N}, h \geq 1$ let $\mathcal{P}^{h}(Y, \mathcal{A})$ denote the $\mathcal{A}$-module of locally polynomial functions of degree $<h$ of the variable $y_{p}: Y \rightarrow \mathbb{Z}_{p}^{\times} \hookrightarrow \mathcal{A}^{\times}$; in particular,

$$
\mathcal{P}^{1}(Y, \mathcal{A})=\mathrm{e}^{\text {loc-const }}(Y, \mathcal{A})
$$

(the $\mathcal{A}$-submodule of locally constant functions). Let also denote $\operatorname{Cloc-an}(Y, \mathcal{A})$ the $\mathcal{A}$-module of locally analytic functions, so that

$$
\mathcal{P}^{1}(Y, \mathcal{A}) \subset \mathcal{P}^{h}(Y, \mathcal{A}) \subset \mathfrak{e}^{l o c-a n}(Y, \mathcal{A}) \subset \mathcal{C}(Y, \mathcal{A}) .
$$

b) Let $V$ be a normed $\mathcal{A}$-module with the norm $|\cdot|_{p, V}$. For a given positive integer $h$ an $h$-admissible measure on $Y$ with values in $V$ is an $\mathcal{A}$-module homomorphism

$$
\tilde{\Phi}: \mathcal{P}^{h}(Y, \mathcal{A}) \rightarrow V
$$

such that for fixed $a \in Y$ and for $v \rightarrow \infty$ the following growth condition is satisfied:

$$
\begin{aligned}
& \left|\int_{a+\left(N p^{v}\right)}\left(y_{p}-a_{p}\right)^{h^{\prime}} d \tilde{\Phi}\right|_{p, V}=o\left(p^{-v\left(h^{\prime}-h\right)}\right) \\
& \text { for all } h^{\prime}=0,1, \ldots, h-1, a_{p}:=y_{p}(a)
\end{aligned}
$$

The condition (7.17) allows to integrate the locally-analytic functions on $Y$ along $\tilde{\Phi}$ using Taylor's expansions! This means: there exists a unique extension of $\tilde{\Phi}$ to $\mathrm{e}^{\text {loc-an }}(Y, \mathcal{A}) \rightarrow V$.

7.1. $U_{p}-$ Operator and the method of canonical projection. We refer to Section 5 of [PaTV] for similar constructions in the case of the $L$-function of one Coleman's family. 
Using the canonical projection $\pi_{\lambda}$. We construct our $H$-admissible measure $\widetilde{\Phi}^{\lambda}$ : $\mathcal{P}^{H}(Y, \mathcal{A}) \rightarrow \mathcal{M}(\mathcal{A})$ out of a sequence of distributions $\Phi_{r}: \mathcal{P}^{1}(Y, \mathcal{A}) \rightarrow \mathcal{M}(\mathcal{A})$ defined on local monomials $y_{p}^{r}$ of each degree $r$ by the rule

$$
\int_{Y} \chi y_{p}^{r} d \widetilde{\Phi}^{\lambda}=\pi_{\lambda}(\tilde{\mathcal{E}}(-r, \chi)), \text { where } \tilde{\mathcal{E}}(-r, \chi) \in M=\mathcal{M}(\mathcal{A}) .
$$

Here $\tilde{\mathcal{E}}(-r, \chi)$ takes values in an $\mathcal{A}$-module

$$
M=\mathcal{M}(\mathcal{A}) \subset \mathcal{A} \llbracket q_{1}, q_{2}, q_{3} \rrbracket\left[R_{1}, R_{2}, R_{3}\right]
$$

of nearly holomorphic (overconvergent) triple modular forms over $\mathcal{A}$ (for $0 \leq r \leq$ $\left.H-1, H=\left[2 \operatorname{ord}_{p} \lambda_{p}\right]+1\right)$, and the formal series $\tilde{\mathcal{E}}(-r, \chi)$ was constructed in the proof of 1) of Main Theorem.

Definition of the canonical projection $\pi_{\lambda}$. Here $\mathcal{A}$ is an $\mathbb{C}_{p}$-algebra, and $\lambda \in \mathcal{A}^{\times}$is a fixed non-zero eigenvalue of triple Atkin's operator $U_{T}=U_{T, p}$, acting on $\mathcal{M}(\mathcal{A})$,

$$
\pi_{\lambda}: \mathcal{M}(\mathcal{A}) \rightarrow \mathcal{M}(\mathcal{A})^{\lambda}
$$

is the canonical projection operator onto the maximal $\mathcal{A}$-submodule $\mathcal{M}(\mathcal{A})^{\lambda}$ over which the operator $U_{T}-\lambda I$ is nilpotent (we call $\mathcal{M}(\mathcal{A})^{\lambda}$ the $\lambda$-characteristic submodule of $\mathcal{M}(\mathcal{A})$ ).

The projector $\pi_{\lambda}$ is defined by its kernel:

$$
\operatorname{Ker} \pi_{\lambda}:=\bigcap_{n \geq 1} \operatorname{Im}\left(U_{T}-\lambda I\right)^{n}, \quad \operatorname{Im} \pi_{\lambda}:=\bigcup_{n \geq 1} \operatorname{Ker}\left(U_{T}-\lambda I\right)^{n} .
$$

\section{TRIPLE MODULAR FORMS}

Triple modular forms are certain formal series

$$
\begin{aligned}
& g=\sum_{n_{1}, n_{2}, n_{3}=0}^{\infty} a\left(n_{1}, n_{2}, n_{3} ; R_{1}, R_{2}, R_{3}\right) q_{1}^{n_{1}} q_{2}^{n_{2}} q_{3}^{n_{3}} \\
& \in \mathcal{A} \llbracket q_{1}, q_{2}, q_{3} \rrbracket\left[R_{1}, R_{2}, R_{3}\right], \text { where } z_{j}=x_{j}+i y_{j} \in \mathbb{H}, R_{j}=\left(4 \pi y_{j}\right)^{-1},
\end{aligned}
$$

with the property that for $\mathcal{A}=\mathbb{C}$, the series converges to a $\mathbb{C}^{\infty}$-modular form on $\mathbb{H}^{3}$ of a given weight $\left(k_{1}, k_{2}, k_{3}\right)$ and character $\left(\psi_{1}, \psi_{2}, \psi_{3}\right), j=1,2,3$. The coefficients $a\left(n_{1}, n_{2}, n_{3} ; R_{1}, R_{2}, R_{3}\right)$ are polynomials in $\mathcal{A}\left[R_{1}, R_{2}, R_{3}\right]$, and the triple Atkin's operator is given by

$U_{T}(g)=\sum_{n_{1}, n_{2}, n_{3}=0}^{\infty} a\left(p n_{1}, p n_{2}, p n_{3} ; p R_{1}, p R_{2}, p R_{3}\right) q_{1}^{n_{1}} q_{2}^{n_{2}} q_{3}^{n_{3}}$.

\section{Eigenfunctions of $U_{p}$ and of $U_{p}^{*}$.}


Functions $f_{j, 0}$ and $f_{j}^{0}$. Recall that for any primitive cusp eigenform $f_{j}=\sum_{n=1}^{\infty} a_{n}(f) q^{n}$, there is an eigenfunction $f_{j, 0}=\sum_{n=1}^{\infty} a_{n}\left(f_{j, 0}\right) q^{n} \in \overline{\mathbb{Q}} \llbracket q \rrbracket$ of $U=U_{p}$ with the eigenvalue $\alpha=\alpha_{p, j}^{(1)} \in \overline{\mathbb{Q}}\left(U\left(f_{0}\right)=\alpha f_{0}\right)$ given by

$$
\begin{aligned}
& f_{j, 0}=f_{j}-\alpha_{p, j}^{(2)} f_{j}\left|V_{p}=f_{j}-\alpha_{p, j}^{(2)} p^{-k / 2} f_{j}\right|\left(\begin{array}{ll}
p & 0 \\
0 & 1
\end{array}\right) \\
& \sum_{n=1}^{\infty} a_{n}\left(f_{j, 0}\right) n^{-s}=\sum_{\substack{n=1 \\
p \nmid n}}^{\infty} a_{n}\left(f_{j}\right) n^{-s}\left(1-\alpha_{p, j}^{(1)} p^{-s}\right)^{-1} .
\end{aligned}
$$

Moreover, there is an eigenfunction $f_{j}^{0}$ of $U_{p}^{*}$ given by

$$
f_{j}^{0}=\left.f_{j, 0}^{\rho}\right|_{k}\left(\begin{array}{cc}
0 & -1 \\
N p & 0
\end{array}\right) \text {, where } f_{j, 0}^{\rho}=\sum_{n=1}^{\infty} \overline{a\left(n, f_{0}\right)} q^{n} .
$$

Therefore, $U_{T}\left(f_{1,0} \otimes f_{2,0} \otimes f_{3,0}\right)=\lambda\left(f_{1,0} \otimes f_{2,0} \otimes f_{3,0}\right)$.

\section{Critical values of the $L$ Function $L\left(f_{1} \otimes f_{2} \otimes f_{3}, s, \chi\right)$}

(compare with Section 7 in [PaTV]).

Choice of Dirichlet characters. For an arbitrary Dirichlet character $\chi \bmod N p^{v}$ consider the following Dirichlet characters:

$$
\begin{aligned}
& \chi_{1} \bmod N p^{v}=\chi, \chi_{2} \bmod N p^{v}=\psi_{2} \bar{\psi}_{3} \chi, \\
& \chi_{3} \bmod N p^{v}=\psi_{1} \bar{\psi}_{3} \chi, \boldsymbol{\psi}=\chi^{2} \psi_{1} \psi_{2} \bar{\psi}_{3} ;
\end{aligned}
$$

later on we impose the condition that the conductors of the corresponding primitive characters $\chi_{0,1}, \chi_{0,2}, \chi_{0,3}$ are $N p$-completes (i.e. have the same prime divisors as resp. those of $N p$ ).

Theorem A (algebraic Properties of the triple Product). Assume that $k_{2}+k_{3}-k_{1} \geq 2$, then for all pairs $(\chi, r)$ such that the corresonding Dirichlet characters $\chi_{j}$ have $N p$-complete conductors, and $0 \leq r \leq k_{2}+k_{3}-k_{1}-2$, we have that

$$
\frac{\Lambda\left(f_{1}^{\rho} \otimes f_{2}^{\rho} \otimes f_{3}^{\rho}, k_{2}+k_{3}-2-r, \psi_{1} \psi_{2} \chi\right)}{\left\langle f_{1}^{\rho} \otimes f_{2}^{\rho} \otimes f_{3}^{\rho}, f_{1}^{\rho} \otimes f_{2}^{\rho} \otimes f_{3}^{\rho}\right\rangle_{T}} \in \overline{\mathbb{Q}}
$$

where

$$
\begin{aligned}
& \left\langle f_{1}^{\rho} \otimes f_{2}^{\rho} \otimes f_{3}^{\rho}, f_{1}^{\rho} \otimes f_{2}^{\rho} \otimes f_{3}^{\rho}\right\rangle_{T}:=\left\langle f_{1}^{\rho}, f_{1}^{\rho}\right\rangle_{N}\left\langle f_{2}^{\rho}, f_{2}^{\rho}\right\rangle_{N}\left\langle f_{3}^{\rho}, f_{3}^{\rho}\right\rangle_{N} \\
& \quad=\left\langle f_{1}, f_{1}\right\rangle_{N}\left\langle f_{2}, f_{2}\right\rangle_{N}\left\langle f_{3}, f_{3}\right\rangle_{N} .
\end{aligned}
$$




\section{Theorems B-D}

Recall: the p-adic weight space and the Mellin transform. (for generalities we also refer to the introduction of [PaTV] in the case of the $L$-function of one Coleman's family). The $p$-adic weight space is the group $X=\operatorname{Hom}_{\text {cont }}\left(Y, \mathbb{C}_{p}^{\times}\right)$of (continuouos) $p$-adic characters of the commutative profinite group $Y=\underset{\leftarrow}{\lim }\left(\mathbb{Z} / N p^{v} \mathbb{Z}\right)^{*}$

The group $X$ is isomorphic to a finite union of $\operatorname{discs} U=\left\{\left.z \in \mathbb{C}_{p}|| z\right|_{p}<1\right\}$.

A $p$-adic $L$-function $L_{(p)}: X \rightarrow \mathbb{C}_{p}$ is a certain meromorphic function on $X$. Such a function usually come from a $p$-adic measure $\mu$ on $Y$ (bounded or admissible in the sense of Amice-Vélu, see $[\mathrm{Am}-\mathrm{V}]$ ). The $p$-adic Mellin transform of $\mu$ is given for all $x \in X$ by

$$
L_{(p)}(x)=\int_{Y_{N, p}} x(y) \mathrm{d} \mu(y), L_{(p)}: X \rightarrow \mathbb{C}_{p}
$$

Theorem $B$ (on admissible measures attached to the triple product:fixed balanced weights case). Under the assumptions as above there exist a $\mathbb{C}_{p}$-valued measure $\tilde{\mu}_{f_{1} \otimes f_{2} \otimes f_{3}}^{\lambda}$ on $Y_{N, p}$, and a $\mathbb{C}_{p}$-analytic function

$\mathcal{D}_{(p)}\left(x, f_{1} \otimes f_{2} \otimes f_{3}\right): X_{p} \rightarrow \mathbb{C}_{p}$, given for all $x \in X_{N, p}$ by the integral $\mathcal{D}_{(p)}\left(x, f_{1} \otimes\right.$ $\left.f_{2} \otimes f_{3}\right)=\int_{Y_{N, p}} x(y) \mathrm{d} \tilde{\mu}_{f_{1} \otimes f_{2} \otimes f_{3}}^{\lambda}(y)$, and having the following properties:

(i) for all pairs $(r, \chi)$ such that $\chi \in X_{N, p}^{\text {tors }}$, and all three corresponding Dirichlet characters $\chi_{j}$ have $N p$-complete conductor $(j=1,2,3)$, and $r \in \mathbb{Z}$ is an integer with $0 \leq r \leq k_{2}+k_{3}-k_{1}-2$, the following equality holds:

$$
\mathcal{D}_{(p)}\left(\chi x_{p}^{r}, f_{1} \otimes f_{2} \otimes f_{3}\right)=i_{p}\left(\frac{\left(\psi_{1} \psi_{2}\right)(2) C_{\chi}^{4\left(k_{2}+k_{3}-2-r\right)}}{G\left(\chi_{1}\right) G\left(\chi_{2}\right) G\left(\chi_{3}\right) G\left(\psi_{1} \psi_{2} \chi_{1}\right) \lambda_{p}^{2 v}}\right.
$$

$\left.\frac{\Lambda\left(f_{1}^{\rho} \otimes f_{2}^{\rho} \otimes f_{3}^{\rho}, k_{2}+k_{3}-2-r, \psi_{1} \psi_{2} \chi\right)}{\left\langle f_{1}^{0} \otimes f_{2}^{0} \otimes f_{3}^{0}, f_{1,0} \otimes f_{2,0} \otimes f_{3,0}\right\rangle_{T, N p}}\right)$

where $v=\operatorname{ord}_{p}\left(C_{\chi}\right), G(\chi)$ denotes the Gauß sum of a primitive Dirichlet character $\chi_{0}$ attached to $\chi$ (modulo the conductor of $\chi_{0}$ ),

(ii) if $\operatorname{ord}_{p} \lambda_{p}=0$ then the holomorphic function in (i) is a bounded $\mathbb{C}_{p}$-analytic function;

(iii) in the general case (but assuming that $\lambda_{p} \neq 0$ ) the holomorphic function in (i) belongs to the type $o\left(\log \left(x_{p}^{H}\right)\right)$ with $H=\left[2 \operatorname{ord}_{p} \lambda_{p}\right]+1$ and it can be represented as the Mellin transform of the $H$-admissible $\mathbb{C}_{p}$-valued measure $\tilde{\mu}_{f_{1} \otimes f_{2} \otimes f_{3}}^{\lambda}$ (in the sense of Amice-Vélu) on $Y$

(iv) Let $k=k_{2}+k_{3}-k_{1} \geq 2$. If $H \leq k-2$ then the function $\mathcal{D}_{(p)}$ is uniquely 
determined by the above conditions (i). Let us describe now $p$-adic measures attached to Garrett's triple product of three Coleman's families

$$
k_{j} \mapsto\left\{f_{j, k_{j}}=\sum_{n=1}^{\infty} a_{n, j}(k) q^{n}\right\}(j=1,2,3) .
$$

Consider the product of three eigenvalues:

$$
\lambda=\lambda_{p}\left(k_{1}, k_{2}, k_{3}\right)=\alpha_{p, 1}^{(1)}\left(k_{1}\right) \alpha_{p, 2}^{(1)}\left(k_{2}\right) \alpha_{p, 3}^{(1)}\left(k_{3}\right)
$$

and assume that the slope of this product

$$
\sigma=\operatorname{ord}_{p}\left(\lambda\left(k_{1}, k_{2}, k_{3}\right)\right)=\sigma\left(k_{1}, k_{2}, k_{3}\right)=\sigma_{1}+\sigma_{2}+\sigma_{3}
$$

is constant and positive for all triplets $\left(k_{1}, k_{2}, k_{3}\right)$ in an appropriate $p$-adic neighbourhood of the fixed triplet of weights $\left(k_{1}, k_{2}, k_{3}\right)$.

Let $\mathcal{A}=\mathcal{A}(\mathcal{B})$ denote an affinoid algebra $\mathcal{A}$ associated with an analytic space $\mathcal{B}=\mathcal{B}_{1} \times \mathcal{B}_{2} \times \mathcal{B}_{3}$, a neighbourhood around $\left(k_{1}, k_{2}, k_{3}\right) \in X^{3}$ (with a given $k$ and $\psi \bmod N)$.

Theorem $C$ (on p-adic measures for families of triple products). Put $H=$ $\left[\operatorname{2ord}_{p}(\lambda)\right]+1$. There exists a sequence of distributions $\Phi_{r}$ on $Y$ with values in $\mathcal{M}=\mathcal{M}(\mathcal{A})$ giving an $H$-admissible measure $\tilde{\Phi}^{\lambda}$ with values in $\mathcal{M}^{\lambda} \subset \mathcal{M}$ with the following properties:

There exists an $\mathcal{A}$-linear form $\ell=\ell_{\boldsymbol{f}_{1} \otimes \boldsymbol{f}_{2} \otimes \boldsymbol{f}_{3}, \lambda}: \mathcal{M}(\mathcal{A})^{\lambda} \rightarrow \mathcal{A}$ (given by 11.24), such that the composition

$$
\tilde{\mu}=\tilde{\mu}_{\boldsymbol{f}_{1} \otimes \boldsymbol{f}_{2} \otimes \boldsymbol{f}_{3}, \lambda}:=\ell_{\boldsymbol{f}_{1} \otimes \boldsymbol{f}_{2} \otimes \boldsymbol{f}_{3}, \lambda}\left(\tilde{\Phi}^{\lambda}\right)
$$

is an $H$-admissible measure with values in $\mathcal{A}$, and for all $\left(k_{1}, k_{2}, k_{3}\right)$ in the affinoid neighborhood $\mathcal{B}=\mathcal{B}_{1} \times \mathcal{B}_{2} \times \mathcal{B}_{3}$, as above, satisfying $k_{1} \leq k_{2}+k_{3}-2$ we have that the evaluated integrals

$$
e v_{\left(k_{1}, k_{2}, k_{3}\right)}\left(\left(\ell_{\boldsymbol{f}_{1} \otimes \boldsymbol{f}_{2} \otimes \boldsymbol{f}_{3}, \lambda}\right)\left(\tilde{\Phi}^{\lambda}\right)\left(y_{p}^{r} \chi\right)\right)
$$

on the arithmetical chracters $y_{p}^{r} \chi$ coincide with the critical special values

$$
\Lambda^{*}\left(f_{1, k_{1}} \otimes f_{2, k_{2}} \otimes f_{3, k_{3}}, k_{2}+k_{3}-2-r, \chi\right)
$$

for $r=0, \cdots, k_{2}+k_{3}-k_{1}-2$, and for all Dirichlet characters $\chi \bmod N p^{v}, v \geq 1$, with all three corresonding Dirichlet characters $\chi_{j}$ given by (6.16), having $N p$ complete conductors. Here the normalisation of $\Lambda^{*}$ includes at the same time certain Gauss sums, Petersson scalar products, powers of $\pi$ and of $\lambda\left(k_{1}, k_{2}, k_{3}\right)$, and a certain finite Euler product. The precise form of the Euler-like $p$-factor is given by a general motivic setting as in [Pa94, [Co, Co-PeRi]; we also refer to Section 7 of [PaTV] in the case of the $L$-function of one Coleman's family. However, our modular construction of the admissible measures of Theorem $\mathrm{C}$ does not use these explicit formulae. Moreover, these measures are uniquely 
determined by general unicity properties by all but a finite number of values on characters $y_{p}^{r} \chi$.

The p-adic Mellin transform and four variable p-adic analytic functions. Any $h$ admissible measure $\tilde{\mu}$ on $Y$ with values in a $p$-adic Banach algebra $\mathcal{A}$ can be caracterized its Mellin transform $\mathcal{L}_{\tilde{\mu}}(x) \mathcal{L}_{\tilde{\mu}}: X \rightarrow \mathcal{A}$, defined by $\mathcal{L}_{\tilde{\mu}}(x)=\int_{Y} x(y) d \tilde{\mu}(y)$, where $x \in X, \mathcal{L}_{\tilde{\mu}}(x) \in \mathcal{A}$,

Key property of $h$-admissible measures $\tilde{\mu}$ : its Mellin transform $\mathcal{L}_{\tilde{\mu}}$ is analytic with values in $\mathcal{A}$.

Let $\mathcal{A}=\mathcal{A}(\mathcal{B})=\mathcal{A}_{1} \hat{\otimes} \mathcal{A}_{2} \hat{\otimes} \mathcal{A}_{3}=\mathcal{A}\left(\mathcal{B}_{1}\right) \hat{\otimes} \mathcal{A}\left(\mathcal{B}_{2}\right) \hat{\otimes} \mathcal{A}\left(\mathcal{B}_{3}\right)$ denote again the Banach algebra $\mathcal{A}$ where $\mathcal{B}$ is an affinoid neighbourhood around $\left(k_{1}, k_{2}, k_{3}\right) \in X^{3}$ (with a given integer $k$ and Dirichlet character $\psi \bmod N$ ).

Theorem $D$ (on p-adic analytic function in four variables). Put $H=\left[2 \operatorname{ord}_{p}(\lambda)\right]+$ 1. There exists a $p$-adic analytic function in four variables $(x, s) \in X \times \mathcal{B}_{1} \times$ $\mathcal{B}_{2} \times \mathcal{B}_{3} \subset X \times X \times X \times X$ :

$$
\mathcal{L}_{\tilde{\mu}}:(x, s) \longmapsto e v_{s}\left(\mathcal{L}_{\tilde{\mu}(x)}\right) \quad\left(x \in X, \quad \mathcal{L}_{\tilde{\mu}}(x) \in \mathcal{A}\right) .
$$

with values in $\mathbb{C}_{p}$, such that for all $\left(k_{1}, k_{2}, k_{3}\right)$ in the affinoid neighborhood as above $\mathcal{B}=\mathcal{B}_{1} \times \mathcal{B}_{2} \times \mathcal{B}_{3}$, satisfying $k_{1} \leq k_{2}+k_{3}-2$, we have that the special values $\mathcal{L}_{\tilde{\mu}}(x, s)$ at the arithmetical chracters $x=y_{p}^{r} \chi$, and $s=\left(k_{1}, k_{2}, k_{3}\right) \in \mathcal{B}$ coincide with the normalized critical special values

$$
L^{*}\left(f_{1, k_{1}} \otimes f_{2, k_{2}} \otimes f_{3, k_{3}}, k_{2}+k_{3}-2-r, \chi\right) \quad\left(r=0, \cdots, k_{2}+k_{3}-k_{1}-2\right),
$$

for Dirichlet characters $\chi \bmod N p^{v}, v \geq 1$, such that all three corresonding Dirichlet characters $\chi_{j}$ given by (6.16), have $N p$-complete conductors where the same normalisation of $L^{*}$ as in Theorem $C$.

Moreover, for any fixed $s=\left(k_{1}, k_{2}, k_{3}\right) \in \mathcal{B}$ the function

$$
x \longmapsto \mathcal{L}_{\tilde{\mu}}(x, s)
$$

is $p$-adic analytic of type $o\left(\log ^{H}(\cdot)\right)$.

Indeed, we obtain the function in question $\mathcal{L}_{\mu}(x, \mathbf{s})$ by evaluation at

$$
\mathbf{s}=\left(\left(s_{1}, \psi_{1}\right),\left(s_{2}, \psi_{2}\right),\left(s_{3}, \psi_{3}\right)\right) \in \mathcal{B}:
$$

this is a $p$-adic analytic function in four variables $(x, \mathbf{s}) \in X \times \mathcal{B}_{1} \times \mathcal{B}_{2} \times \mathcal{B}_{3} \subset$ $X \times X \times X \times X$ :

$$
\mathcal{L}_{\tilde{\mu}}(x, \mathbf{s}):=e v_{\mathbf{s}}\left(\mathcal{L}_{\tilde{\mu}}\right)(x) \quad\left(x \in X, \mathbf{s} \in \mathcal{B}_{1} \times \mathcal{B}_{2} \times \mathcal{B}_{3}, \mathcal{L}_{\tilde{\mu}}(x) \in \mathcal{A}\right) .
$$

This is a joint work in progress with S.Boecherer, we use:

1) the higher twists of the Siegel-Eisenstein series, introduced in [Boe-Schm, 
2) Ibukiyama's differential operators (see [Ibu], [BSY]).

\section{SCheme of the Proof}

\subsection{Boecherer's higher twist.}

Boecherer's Higher Twist. 1) We define the higher twist of the series $F_{\chi, r}=$ $\sum_{\mathcal{T}} a_{\chi, r}(R, \mathcal{T}) q^{\mathcal{T}}$ by some Dirichlet characters $\bar{\chi}_{1}, \bar{\chi}_{2}, \bar{\chi}_{3}$ as the following formal nearly holomorphic Fourier expansion:

$$
F_{\chi, r}=\sum_{\mathcal{T}} \bar{\chi}_{1}\left(t_{12}\right) \bar{\chi}_{2}\left(t_{13}\right) \bar{\chi}_{3}\left(t_{23}\right) a_{\chi, r}(R, \mathcal{T}) q^{\mathcal{T}}
$$

The seies 11.22 is a Siegel modular form of some higher level, but it has additional symmetries with respect to symplectic embedding $\iota_{3}: \Gamma_{0}\left(N p^{2 v}\right) \times$ $\Gamma_{0}\left(N p^{2 v}\right) \times \Gamma_{0}\left(N p^{2 v}\right) \rightarrow \mathrm{Sp}_{3}$ : its triple Nebentypus character does not depend on $\chi \bmod N p^{v}$, and is equal to $\left(\psi_{1}, \psi_{2}, \psi_{3}\right)$, if we choose Dirichlet characters as in 6.16$)$ :

$$
\begin{aligned}
& \chi_{1} \bmod N p^{v}=\chi, \chi_{2} \bmod N p^{v}=\psi_{2} \bar{\psi}_{3} \chi \\
& \chi_{3} \bmod N p^{v}=\psi_{1} \bar{\psi}_{3} \chi, \boldsymbol{\psi}=\chi^{2} \psi_{1} \psi_{2} \bar{\psi}_{3} .
\end{aligned}
$$

We use the Siegel-Eisenstein series $F_{\chi, r}$ which depends on the character $\chi$, but its precise nebentypus character is $\boldsymbol{\psi}=\chi^{2} \psi_{1} \psi_{2} \bar{\psi}_{3}$, and it is defined by $F_{\chi, r}=$ $G^{\star}\left(z,-r ; k,\left(N p^{v}\right)^{2}, \boldsymbol{\psi}\right)$, where $z$ denotes a variable in the Siegel upper half space $\mathbb{H}_{3}$, and the normalized series $G^{\star}\left(z, s ; k,\left(N p^{v}\right)^{2}, \boldsymbol{\psi}\right)$ is given by 5.11).

This series depends on $s=-r$, and for the critical values at integral points $s \in \mathbb{Z}$ such that $2-k \leq s \leq 0$, it represents a (nearly) holomorphic Siegel modular form in the sense of Shimura [ShiAr]:

$$
F_{\chi, r}=\sum_{\mathcal{T}} \operatorname{det}(\mathcal{T})^{k-2 r-\kappa} Q(R, \mathcal{T} ; k-2 r, r) a_{\chi, r}(\mathcal{T}) q^{\mathcal{T}}
$$

\subsection{Ibukiyama's differential operator.}

Ibukiyama's differential operator. 2) We use an algebraic version of Ibukiyama's differential operator, which generalizes the algebraic "pull-back": it transforms a nearly holomorphic Siegel modular form of weight $k$

to a nearly holomorphic triple modular form of weight $\left(k_{1}, k_{2}, k_{3}\right)\left(k=k_{2}+\right.$ $\left.k_{3}-k_{1}\right)$. 

form

On a holomorphic Siegel modular form $F=\sum_{\mathcal{T}} a(\mathcal{T}) q^{\mathcal{T}}$, this operator has the

$$
\mathcal{L}_{k}^{\lambda, \nu}(F)=\sum_{\mathcal{T}} \mathcal{P}\left(k_{1}, k_{2}, k_{3}, 0, \mathcal{T}\right) a(\mathcal{T}) q_{1}^{t_{11}} q_{2}^{t_{22}} q_{3}^{t_{33}}
$$

where $\lambda=k_{1}-k_{3} \geq \mu=k_{1}-k_{2} \geq 0$, and $\mathcal{P}\left(k_{1}, k_{2}, k_{3} ; r ; \mathcal{T}\right)$ is certain Ibukiyama's polynomial, equal to $\left(t_{11} t_{22} t_{33}\right)^{\lambda}\left(t_{12} t_{13} t_{23}\right)^{\mu}$, if $r=0$.

- As a result we obtain a sequence of triple modular distributions $\Phi_{r}(\chi)$ with values in the tensor product $\mathcal{M}_{T}(\mathcal{A})=\mathcal{M}(\mathcal{A}) \widehat{\otimes}_{\mathcal{A}} \mathcal{N}(\mathcal{A}) \widehat{\otimes}_{\mathcal{A}} \mathcal{M}(\mathcal{A})$ of three Banach $\mathcal{A}$-modules of arithmetical nearly holomorphic modular forms (the normalizing factor $2^{r}$ is neeeded in order to prove certain congruences between $\Phi_{r}$ ). Note that $\mathcal{M}_{T}(\mathcal{A})$ is again a Banach $\mathcal{A}$-module on which $U_{T}$ acts as a completely continuous operator.

The important property of the triple modular forms $\Phi_{r}(\chi)$ : the nebentypus character is fixed and is equal to $\left(\psi_{1}, \psi_{2}, \psi_{3}\right)$ (for all $\left(k_{1}, k_{2}, k_{3}\right)$ and $\chi$ in question).

Using this property we compute the canonical projection $\pi_{\lambda}\left(\Phi_{r}(\chi)\right)$ of the triple modular form $\Phi_{r}(\chi)$ onto the $\lambda$-characteristic $\mathcal{A}$-submodule $\mathcal{M}_{T}{ }^{\lambda}(\mathcal{A})$ of the triple Atkin's operator $U_{T, p}$ :

$$
\pi_{\lambda}: \mathcal{M}_{T}(\mathcal{A}) \rightarrow \mathcal{M}_{T}^{\lambda}(\mathcal{A})
$$

- We prove that the resulting sequence of modular distributions $\pi_{\lambda}\left(\Phi_{r}\right)$ on the profinite group $Y$ produces a certain $p$-adic admissible measure $\tilde{\Phi}^{\lambda}$ (in the sense of Amice-Vélu, $[\mathrm{Am}-\mathrm{V}$ ]) with values in a certain locally free $\mathcal{A}$-submodule of finite rank

$$
\mathcal{M}_{T}^{\lambda}(\mathcal{A}) \subset \mathcal{M}_{T}(\mathcal{A}) \subset \bigcup_{v \geq 0} \mathcal{M}_{T}\left(N p^{v}, \psi_{1}, \psi_{2}, \psi_{3} ; \mathcal{A}\right)
$$

of formal nearly holomorphic triple modular forms of all levels $N p^{v}$ and the fixed nebentypus characters $\left(\psi_{1}, \psi_{2}, \psi_{3}\right)$. We use congruences between triple modular forms $\Phi_{r}(\chi) \in \mathcal{M}_{T}(\mathcal{A})$ (they have explicit formal Fourier coefficients).

Then we use a general admissibility criterion saying that these congruences imply $H$-admissibility for their projections in $\mathcal{M}_{T}^{\lambda}(\mathcal{A})$, where $H=\left[2 \operatorname{ord}_{p}(\lambda)\right]+1$.

11.3. Algebraic linear form. We refer here to Section 6 of [PaTV] for similar constructions in the case of the $L$-function of one Coleman's family. 
. 3) From $\mathcal{M}_{T}^{\lambda}(\mathcal{A})$ to $\mathcal{A}$ : we use a $\overline{\mathbb{Q}}$-valued linear forms of type

$$
\mathcal{L}: h \longmapsto \frac{\left\langle f_{1}^{0} \otimes f_{2}^{0} \otimes f_{3}^{0}, h\right\rangle}{\left\langle f_{1}^{0}, f_{1,0}\right\rangle\left\langle f_{2}^{0}, f_{2,0}\right\rangle\left\langle f_{3}^{0}, f_{3,0}\right\rangle}
$$

where $f_{j}^{0}$ is the eigenfunction 8.18 of the conjugate Atkin's operator $U_{p}^{*}$, and $f_{j, 0}$ is the eigenfunction 8.19 of $U_{p}$. The linear form $\mathcal{L}$ is defined on the finite dimensional $\overline{\mathbb{Q}}$-vector characteristic subspace

$$
\begin{aligned}
& h \in \mathcal{M}_{\mathbf{k}}(\overline{\mathbb{Q}})^{\lambda(\mathbf{k})} \subset \\
& \mathcal{M}_{k_{1}, r^{*}}\left(N p, \psi_{1} ; \overline{\mathbb{Q}}\right) \otimes \mathcal{M}_{k_{2}, r^{*}}\left(N p, \psi_{2} ; \overline{\mathbb{Q}}\right) \otimes \mathcal{M}_{k_{3}, r^{*}}\left(N p, \psi_{3} ; \overline{\mathbb{Q}}\right) .
\end{aligned}
$$

This map is then extended to an $\mathcal{A}$-linear map

$$
\ell=\ell_{\mathbf{f}_{1} \otimes \mathbf{f}_{2} \otimes \mathbf{f}_{3}, \lambda}: \mathcal{M}(\mathcal{A})^{\lambda} \rightarrow \mathcal{A} ;
$$

on the locally free $\mathcal{A}$-module of finite rank $\mathcal{M}(\mathcal{A})^{\lambda}$.

- This map produces a sequence of $\mathcal{A}$-valued distributions $\mu_{r}^{\lambda}(\chi) \in \mathcal{A}$ in such a way that for all suitable weights $\mathbf{k} \in \mathcal{B}$ one has

$$
e v_{\mathbf{k}}\left(\mu_{r}^{\lambda}(\chi)\right)=\mathcal{L}\left(e v_{\mathbf{k}}\left(\pi_{\lambda}\left(\Phi_{r}\right)(\chi)\right)\right), \lambda \in \mathcal{A}^{\times}, \lambda(\mathbf{k}) \in \overline{\mathbb{Q}}^{\times},
$$

where $\mathbf{k}=\left(k_{1}, k_{2}, k_{3}\right) \in \mathcal{B}, e v_{\mathbf{k}}: \mathcal{B} \rightarrow \mathbb{C}_{p}$ denotes the evaluation map with the property

$$
e v_{\mathbf{k}}: \mathcal{M}(\mathcal{A}) \rightarrow \mathcal{M}_{\mathbf{k}}\left(\mathbb{C}_{p}\right)
$$

. More precisely, we consider three auxilliary families of modular forms

$$
\begin{aligned}
& \tilde{f}_{j, k_{j}}(z)= \\
& \sum_{n=1}^{\infty} \tilde{a}_{n, j, k_{j}} e(n z) \in S_{k_{j}}\left(\Gamma_{0}\left(N_{j} p^{\nu_{j}}\right), \psi_{j}\right), \quad\left(1 \leq j \leq 3, \nu_{j} \geq 1\right),
\end{aligned}
$$

with the same eigenvalues as those of (10.21),

for all Hecke operators $T_{q}$, with $q$ prime to $N p$. In our construction we use as $\tilde{f}_{j, k_{j}}$ certain "easy transforms" of primitive cusp forms in 1.1. In particular, we choose as $\tilde{f}_{j}$ certain eigenfunctions $\tilde{f}_{j, k_{j}}=f_{j, k_{j}}^{0}$ of the adjoint Atkin's operator $U_{p}^{*}$, in this case we denote by $f_{j, k_{j}, 0}$ the corresponding eigenfunctions of $U_{p}$.

The $\overline{\mathbb{Q}}$-linear form $\mathcal{L}$ produces a $\mathbb{C}_{p}$-valued admissible measure $\tilde{\mu}^{\lambda}=\ell\left(\tilde{\Phi}^{\lambda}\right)$ starting from the modular $p$-adic admissible measure $\tilde{\Phi}^{\lambda}$ of stage 3 ), where $\ell$ : $\mathcal{M}_{T}\left(\mathbb{C}_{p}\right) \rightarrow \mathbb{C}_{p}$ denotes a $\mathbb{C}_{p}$-linear form, interpolating $\mathcal{L}$.

11.4. Evaluation of $p$-adic integrals. We refer to Section 7 of [PaTV] for similar constructions in the case of the $L$-function of one Coleman's family. 
L-values and p-adic integrals. 4) We show that for any appropriate Dirichlet character $\chi \bmod N p^{v}$ the integral

$$
\mu_{r}^{\lambda}(\chi)=\mathcal{L}\left(\pi_{\lambda}\left(\Phi_{r}(\chi)\right)\right) \in \mathcal{A}
$$

evaluated at $\left(k_{1}, k_{2}, k_{3}\right) \in \mathcal{B}=\mathcal{B}_{1} \times \mathcal{B}_{2} \times \mathcal{B}_{3}$, coincides (up to a normalisation) with the special $L$-value

$$
L^{*}\left(f_{1, k_{1}}^{\rho} \otimes f_{2, k_{2}}^{\rho} \otimes f_{3, k_{3}}^{\rho}, k_{2}+k_{3}-2-r, \psi_{1} \psi_{2} \chi\right)
$$

under the above assumptions on $\chi$ and $r$ ).

A general integral representation of Garrett's type. The basic idea how a Dirichlet character $\chi$ is incorporated in the integral representation [Ga87, BoeSP] is somewhat similar to the one used in [Boe-Schm, but (surprisingly) more complicated to carry out.

Note however that the existence of a $\mathcal{A}$-valued admissible measure $\tilde{\mu}^{\lambda}=\ell\left(\tilde{\Phi}^{\lambda}\right)$ established at stage 4), does not depend on this technical computation.

In order to control the denominators of the modular forms

$$
\pi_{\lambda}(\tilde{\mathcal{E}}(-r, \chi)) \in \mathcal{M}^{\lambda}(\mathcal{A})=: \Phi_{r}(\chi)
$$

used in the construction (the admissibility condition) we use the following result.

\section{Criterion of ADmissibility}

TheOREM 12.1 (Criterion of admissibility ). Let $\alpha \in \mathcal{A}^{*}, 0<|\alpha|_{p}<1$ and suppose that there exists a positive integer $\varkappa$ such that the following conditions are satisfied:

1) for all $r=0,1, \cdots, h-1$ with $h=\left[\varkappa_{0} \operatorname{ord}_{p} \alpha\right]+1$, and $v \geq 1$,

$$
\Phi_{r}\left(a+\left(N p^{v}\right)\right) \in \mathcal{M}\left(N p^{\varkappa v}\right) \quad \text { (the level condition) }
$$

2) the following congruence for the coefficients holds: for all $t=0,1, \cdots, h-1$

$$
U^{\varkappa v} \sum_{r=0}^{t}\left(\begin{array}{l}
t \\
r
\end{array}\right)\left(-a_{p}\right)^{t-r} \Phi_{r}\left(a+\left(N p^{v}\right)\right) \equiv 0 \bmod p^{v t}
$$

(the divisibility condition)

Then the linear form given by $\tilde{\Phi}^{\alpha}\left(\delta_{a+\left(N p^{v}\right)} y_{p}^{r}\right):=\pi_{\alpha}\left(\Phi_{r}\left(a+\left(N p^{v}\right)\right)\right.$ on local monomials (for all $r=0,1, \cdots, h-1$ ), is an $h$-admissible measure: $\tilde{\Phi}^{\alpha}$ : $\mathcal{P}^{h}(Y, \overline{\mathbb{Q}}) \rightarrow \mathcal{M}^{\alpha} \subset \mathcal{M}$ 
Proof uses the commutative diagram:

$$
\begin{aligned}
& \mathcal{M}\left(N p^{v+1}, \psi ; \mathcal{A}\right) \stackrel{\pi_{\alpha, v}}{\longrightarrow} \mathcal{M}^{\alpha}\left(N p^{v+1}, \psi ; \mathcal{A}\right) \\
& U^{v} \downarrow \quad \downarrow 2 U^{v} \\
& \mathcal{M}(N p, \psi ; \mathcal{A}) \underset{\pi_{\alpha, 0}}{\longrightarrow} \mathcal{M}^{\alpha}(N p, \psi ; \mathcal{A})=\mathcal{M}^{\alpha}\left(N p^{v+1}, \psi ; \mathcal{A}\right) .
\end{aligned}
$$

The existence of the projectors $\pi_{\alpha, v}$ comes from Coleman's Theorem A.4.3 [CoPB].

On the right: $U$ acts on the locally free $\mathcal{A}$-module $\mathcal{M}^{\alpha}\left(N p^{v+1}, \mathcal{A}\right)$ via the matrix:

$$
\begin{aligned}
& \left(\begin{array}{cccc}
\alpha & \cdots & \cdots & * \\
0 & \alpha & \cdots & * \\
0 & 0 & \ddots & \cdots \\
0 & 0 & \cdots & \alpha
\end{array}\right) \text { where } \alpha \in \mathcal{A}^{\times} \\
& \Longrightarrow U^{v} \text { is an isomorphism over } \mathcal{A},
\end{aligned}
$$

and one controls the denominators of the modular forms of all levels $v$ by the relation:

$$
\pi_{\alpha, v}(h)=U^{-v} \pi_{\alpha, 0}\left(U^{v} h\right)=: \pi_{\alpha}(h)
$$

The equality (12.28) can be used as the definition of $\pi_{\alpha}$ at any level $N p^{v}$.

The growth condition (see 7.17$)$ ) for $\pi_{\alpha}\left(\Phi_{r}\right)$ is deduced from the congruences (12.27) between modular forms, using the relation (12.28). 


\section{REFERENCES}

[Am-V] Amice, Y. and VÉLu, J., Distributions p-adiques associées aux séries de Hecke, Journées Arithmétiques de Bordeaux (Conf. Univ. Bordeaux, 1974), Astérisque no. 24/25, Soc. Math. France, Paris 1975, 119 - 131

[Boe1] Böcherer, S., Über die Funktionalgleichung automorpher L-Funktionen zur Siegelscher Modulgruppe, J. reine angew. Math. 362 (1985) 146-168

[Boe-Ha] Boecherer, S., Heim, B. L-functions for $G S p_{2} \times G l_{2}$ of mixed weights. Math. Z. $235,11-51(2000)$

[Boe-Pa6] S. Böcherer and A.A. Panchishrin, Admissible p-adic measures attached to triple products of elliptic cusp forms, accepted in Documenta Math. in March 2006 (a special volume dedicated to John Coates).

[BSY] Böcherer, S., Satoh, T., and Yamazaki, T., On the pullback of a differential operator and its application to vector valued Eisenstem series, Comm. Math. Univ. S. Pauli, 41 (1992), 1-22.

[Boe-Schm] Böcherer, S., and Schmidt, C.-G., p-adic measures attached to Siegel modular forms, Ann. Inst. Fourier 50, N5, 1375-1443 (2000).

[BoeSP] Böcherer, S. and Schulze-Pillot, R., On the central critical value of the triple product L-function. David, Sinnou (ed.), Number theory. Séminaire de Théorie des Nombres de Paris 1993-94. Cambridge: Cambridge University Press. Lond. Math. Soc. Lect. Note Ser. 235, 1-46 (1996).

[Chand70] Chandrasekharan, K. Arithmetical functions. Berlin-Heidelberg-New York, Springer-Verlag (1970).

[Co] Contes, J. On p-adic L-functions. Sem. Bourbaki, 40eme annee, 1987-88, n 701, Asterisque (1989) 177-178.

[Co-PeRi] Contes, J. and Perrin-Riou, B., On p-adic L-functions attached to motives over $\mathbb{Q}$, Advanced Studies in Pure Math. 17, 23-54 (1989)

[CoPB] Coleman, Robert F., p-adic Banach spaces and families of modular forms, Invent. Math. 127, No.3, 417-479 (1997)

[CoM] Coleman, R. and Mazur, B., The eigencurve, Scholl, A. J. (ed.) et al., Galois representations in arithmetic algebraic geometry. Proceedings of the symposium, Durham, UK, July 9-18, 1996. Cambridge: Cambridge University Press. Lond. Math. Soc. Lect. Note Ser. 254, 1-113 (1998).

[CST98] Coleman, R., Stevens, G., Teitelbaum, J., Numerical experiments on families of padic modular forms, in: Computational perspectives in Number Theory, ed. by D.A. Buell, J.T. Teitelbaum, Amer. Math. Soc., 143-158 (1998).

[Colm03] Colmez, P. La conjecture de Birch et Swinnerton-Dyer p-adique. Séminaire Bourbaki, exposé nº 919, juin 2003.

[Cour] Courtieu, M., Familles d'opérateurs sur les formes modulaires de Siegel et fonctions L $p$-adiques,

http://www-fourier.ujf-grenoble.fr/THESE/ps/ t101.ps.gz, Thèse de Doctorat, Institut Fourier, 2000, 1-122.

[CourPa] Courtieu, M., Panchishkin, A.A., Non-Archimedean L-Functions and Arithmetical Siegel Modular Forms, Lecture Notes in Mathematics 1471, Springer-Verlag, 2004 (2nd augmented ed.)

[De79] Deligne P., Valeurs de fonctions L et périodes d'intégrales, Proc. Symp. Pure Math AMS 33 (part 2) (1979) 313-342.

[Fei86] Feit, P., Poles and Residues of Eisenstein Series for Symplectic and Unitary Groups, Memoir AMS $61 \mathrm{~N}^{\circ} 346$ (1986), 89 p. 
[Ga87] Garrett, P.B., Decomposition of Eisenstein series: Rankin triple products, Ann. of Math. 125 (1987), 209-235

[GaHa] Garrett, P.B. and Harris, M., Special values of triple product L-functions, Amer. J. Math 115 (1993) 159-238

[HaH] HA HuY, KHOAI, p-adic interpolation and the Mellin-Mazur transform, Acta Math. Viet. 5, 77-99 (1980).

[Ka-Za] Kaneko, M., Zagier, Don, A generalized Jacobi theta function and quasimodular forms. The moduli space of curves (Texel Island, 1994), 165-172, Progr. Math., 129, Birkhäuser Boston, Boston, MA, 1995.

[Ha93] Harris, M., and Kudla, S., The central critical value of a triple product L-functions, Ann. Math. 133 (1991), 605-672

[HaTi] Harris, M., and Tilouine, J., p-adic measures and square roots of special values of triple product L-functions, Math. Annalen, 320, N 1 (2001)

[Hi85] HidA, H., A p-adic measure attached to the zeta functions associated with two elliptic cusp forms. I, Invent. Math. 79 (1985) 159-195

[Hi86] HidA, H., Galois representations into $G L_{2}\left(\mathbb{Z}_{p}[[X]]\right)$ attached to ordinary cusp forms, Invent. Math. 85 (1986) 545-613

[Hi90] HidA, H., Le produit de Petersson et de Rankin p-adique, Séminaire de Théorie des Nombres, Paris 1988-1989, 87-102, Progr. Math., 91, Birkhäuser Boston, Boston, MA, 1990.

[JoH05] JoRY-Hugue, F., Unicité des h-mesures admissibles p-adiques données par des valeurs de fonctions $L$ sur les caractères, Prépublication de l'Institut Fourier (Grenoble), $\mathrm{N}^{\circ} 676$, $1-33,2005$

[Ibu] IBUkiyama, T. On differential operators on automorphic forms and invariant pluriharmonic polynomials, Comm. Math. Univ. S. Pauli 48 (1999), 103-118

[Ka76] Katz, N.M., p-adic interpolation of real analytic Eisenstein series, Ann. of Math. 104 (1976) 459-571

[Ka78] Katz, N.M., p-adic L-functions for CM-fields, Invent. Math. 48 (1978) 199-297

[Ku-Le] Kubota T. and Leopoldt H.-W. , Eine p-adische Theorie der Zetawerte, J. reine angew. Math. 214/215 (1964) 328-339

[Maa] MaAss, H., Siegel's Modular Forms and Dirichlet Series, Springer-Verlag, Lect. Notes in Math. $\mathrm{N}^{\circ} 216$ (1971).

[Man73] Manin, Yu.I., Periods of cusp forms and p-adic Hecke series, Mat. Sbornik 92 (1973) 378-401(in Russian); Math. USSR, Sb. 21(1973), 371-393 (1975) (English translation).

[Ma-Pa77] Manin, Yu.I. and Panchishkin, A.A. , Convolutions of Hecke series and their values at integral points, Mat. Sbornik, 104 (1977) 617-651 (in Russian); Math. USSR, Sb. 33, 539-571 (1977) (English translation).

[MTT] Mazur, B., Tate, J. and Teitelbaum, J., On p-adic analogues of the conjectures of Birch and Swinnerton-Dyer, Invent. Math. 84, 1-48 (1986).

[Miy] Miyake, Toshitsune, Modular forms. Transl. from the Japanese by Yoshitaka Maeda. Berlin etc.: Springer-Verlag. viii, 335 p. (1989).

[Or] Orloff T., Special values and mixed weight triple products (with an Appendix by D.Blasius), Invent. Math. 90 (1987) 169-180

[Nag1] NagaokA, S., A remark on Serre's example of p-adic Eisenstein series, Math. Z. 235 (2000) 227-250.

[Nag2] NaGAOKA, S., Note on mod $p$ Siegel modular forms, Math. Z. 235 (2000) 405-420.

[Pa94] Panchishrin, A.A., Admissible Non-Archimedean standard zeta functions of Siegel modular forms, Proceedings of the Joint AMS Summer Conference on Motives, Seattle, July 20-August 2 1991, Seattle, Providence, R.I., 1994, vol.2, 251 - 292 
[PaSE] Panchishkin, A.A., On the Siegel-Eisenstein measure. Israel Journal of Mathematics, Vol. 120, Part B(2000), 467-509

[PaMMJ] Panchishkin, A.A., A new method of constructing p-adic L-functions associated with modular forms, Moscow Mathematical Journal, 2 (2002), Number 2, 1-16

[PaTV] Panchishkin, A.A., Two variable p-adic L functions attached to eigenfamilies of positive slope, Invent. Math. v. 154, N3 (2003), pp. 551 - 615

[PaMMJ2] PAnchishkin, A.A., The Maass-Shimura differential operators and congruences between arithmetical Siegel modular forms, (accepted in Moscow Mathematical Journal in October 2005 (39 p.).

[PSRa] Piatetski-Shapiro, I.I., and Rallis, S., Rankin triple L-functions, Compositio Math. 64 (1987) 333-399

[Se73] Serre, J.-P., Formes modulaires et fonctions zêta p-adiques, Lect Notes in Math. 350 (1973) 191-268 (Springer Verlag)

[SePB] Serre, J.-P., Endomorphisms completement continus des espaces de Banach p-adiques, Publ. Math. Inst. Hautes Etud. Sci., 12, 69-85 (1962).

[Shi71] Shimura G., Introduction to the Arithmetic Theory of Automorphic Functions, Princeton Univ. Press, 1971

[Shi76] Shimura G., The special values of the zeta functions associated with cusp forms. Comm. Pure Appl. Math. 29 (1976), no. 6, 783-804.

[Shi77] Shimura G., On the periods of modular forms, Math. Annalen 229 (1977) 211-221

[Sh83] Shimura, G., On Eisentsein Series, Duke Math. J; 50 (1983), p. 417-476.

[ShiAr] Shimura, G., Arithmeticity in the theory of automorphic forms, Mathematical Surveys and Monographs. 82. Providence, RI: American Mathematical Society (AMS). x, 302 p. (2000)

[Til-U] Tilouine, J. and Urban, E. , Several variable p-adic families of Siegel-Hilbert cusp eigenforms and their Galois representations, Ann. scient. Éc. Norm. Sup. $4^{\mathrm{e}}$ série, 32 (1999) 499-574.

[V] VIsIK, M.M., Non-Archimedean measures connected with Dirichlet series, Math. USSR Sb. 28 (1976), 216-228 (1978).

A. A. Panchishkin

Institut Fourier, Université Grenoble-1

E-mail: Alexei.Pantchichkine@ujf-grenoble.fr 